\title{
Engineering Sensorial Delay to Control Phototaxis and Emergent Collective Behaviors
}

\author{
Mite Mijalkov, ${ }^{1,2}$ Austin McDaniel, ${ }^{3}$ Jan Wehr, ${ }^{3}$ and Giovanni Volpe ${ }^{1,2}$ \\ ${ }^{1}$ Soft Matter Lab, Department of Physics, Bilkent University, Cankaya, 06800 Ankara, Turkey \\ ${ }^{2}$ UNAM-National Nanotechnology Research Center, Bilkent University, Ankara 06800, Turkey \\ ${ }^{3}$ Department of Mathematics and Program in Applied Mathematics, \\ University of Arizona, Tucson, Arizona 85721, USA
}

(Received 7 September 2015; published 29 January 2016)

\begin{abstract}
Collective motions emerging from the interaction of autonomous mobile individuals play a key role in many phenomena, from the growth of bacterial colonies to the coordination of robotic swarms. For these collective behaviors to take hold, the individuals must be able to emit, sense, and react to signals. When dealing with simple organisms and robots, these signals are necessarily very elementary; e.g., a cell might signal its presence by releasing chemicals and a robot by shining light. An additional challenge arises because the motion of the individuals is often noisy; e.g., the orientation of cells can be altered by Brownian motion and that of robots by an uneven terrain. Therefore, the emphasis is on achieving complex and tunable behaviors from simple autonomous agents communicating with each other in robust ways. Here, we show that the delay between sensing and reacting to a signal can determine the individual and collective long-term behavior of autonomous agents whose motion is intrinsically noisy. We experimentally demonstrate that the collective behavior of a group of phototactic robots capable of emitting a radially decaying light field can be tuned from segregation to aggregation and clustering by controlling the delay with which they change their propulsion speed in response to the light intensity they measure. We track this transition to the underlying dynamics of this system, in particular, to the ratio between the robots' sensorial delay time and the characteristic time of the robots' random reorientation. Supported by numerics, we discuss how the same mechanism can be applied to control active agents, e.g., airborne drones, moving in a three-dimensional space. Given the simplicity of this mechanism, the engineering of sensorial delay provides a potentially powerful tool to engineer and dynamically tune the behavior of large ensembles of autonomous mobile agents; furthermore, this mechanism might already be at work within living organisms such as chemotactic cells.
\end{abstract}

DOI: 10.1103/PhysRevX.6.011008

\section{INTRODUCTION}

The interaction between several simple autonomous agents can give rise to complex collective behaviors. This is observed at all scales, from the organization of bacterial colonies [1,2] and the foraging of ants and bees [3] to the assembly of schools of fish [4] and the collective motion of human crowds [5]. Inspired by these natural systems, the same principles have been applied to engineer autonomous robots capable of performing tasks such as search-and-rescue in disaster zones, surveillance of hazardous areas, and targeted object delivery in complex environments [6-11].

Complex behaviors can emerge even if each agent follows very simple rules, senses only its immediate surroundings, and directly interacts only with nearby agents, without having any knowledge of an overall plan [12,13]. For example, while performing their swim-and-tumble motion,

Published by the American Physical Society under the terms of the Creative Commons Attribution 3.0 License. Further distribution of this work must maintain attribution to the author(s) and the published article's title, journal citation, and DOI.
Subject Areas: Interdisciplinary Physics, Soft Matter

chemotactic bacteria are able to climb a chemotactic gradient, e.g., in order to move towards regions rich in nutrients, by simply adjusting their tumbling rate depending on the chemical concentration they sense [2,14]. Furthermore, by releasing chemoattractant molecules into their surroundings, they are capable of generating a chemical gradient around themselves to which other cells can respond, e.g., in order to create bacterial colonies [1]. Similarly, simple mechanisms are at work in the organization of flocks of birds, schools of fish, and herds of mammals, whereby complex collective behaviors result from each animal reacting to signals sent by its neighbors. A similar approach has also been fruitfully explored in order to build artificial systems with robust behaviors arising from interactions between very simple constituent agents [6,10,11,15-18]. Complex behaviors emerging from agents obeying simple rules have the advantage of being extremely robust: For example, even if one or more agents are destroyed, the others can continue to work together to complete the task at hand; agents can also be removed or added midtask without significantly affecting the final result.

Here, we experimentally and theoretically demonstrate that it is possible to engineer the individual and collective 
behavior of autonomous agents whose motion is intrinsically noisy by making use of the delay in their sensorial feedback cycle. In other words, we show how the delay between the time when an agent senses a signal and the time when it reacts to it can be used as a new parameter for the engineering of large-scale organization of autonomous agents. This proposal is inspired by the motion of chemotactic cells, which are able to climb a chemical gradient by adjusting a different parameter, i.e., their tumbling rate, in response to the concentration of molecules in their surroundings. We demonstrate that the collective behavior of a group of phototactic robots, capable of emitting a radially decaying light field, can be tuned from segregation to aggregation and clustering by controlling the delay with which they adjust their propulsion speed to the light intensity. More precisely, we show that this transition occurs as the ratio between the robots' sensorial delay time and characteristic time of their random reorientation crosses a certain critical value.

\section{SINGLE AGENT}

We start by considering a single autonomous agent that moves in a plane and whose orientation is subject to noise. This happens naturally in the case of microswimmersmicroscopic particles capable of self-propulsion such as motile bacteria and cells $[19,20]$ — as the direction of their motion changes randomly over time because of the presence of rotational Brownian motion [2]. Similarly, autonomous robots, animals, and even humans can undergo a random reorientation when moving in the absence of external reference points (a striking example of this is an experiment where blindfolded people who were asked to walk in a straight line spontaneously moved along bent trajectories [21]). Such motion is known as active Brownian motion and can be modeled by the following system of stochastic differential equations [12,20,22,23]:

$$
\left\{\begin{array}{l}
\frac{d x_{t}}{d t}=v \cos \phi_{t}, \\
\frac{d y_{t}}{d t}=v \sin \phi_{t}, \\
\frac{d \phi_{t}}{d t}=\sqrt{\frac{2}{\tau}} \eta_{t},
\end{array}\right.
$$

where $\left(x_{t}, y_{t}\right)$ is the position of the agent in the plane at time $t, \phi_{t}$ is its orientation, $v$ is its speed, $\tau$ is the reorientation characteristic time (i.e., the time after which the standard deviation of the agent's rotation is $1 \mathrm{rad}$ ), and $\eta_{t}$ is a white noise driving the agent's reorientation, as shown in Fig. 1(a). The reorientation time $\tau$ can be associated with an effective reorientation diffusion constant $D_{\mathrm{R}}=\tau^{-1}$, which, in the case of microswimmers, often coincides with the rotational diffusion constant of the particle.

Furthermore, we assume that this agent moves in the presence of an external intensity field to which it reacts by adjusting its speed as a function of the instantaneous intensity it senses. We have realized this experimentally by using a phototactic robot (Elisa-3 [24]) moving within the light gradient generated by a 100-W infrared lamp, which emitted a radially symmetric light intensity radially decaying with a characteristic length $R=35 \mathrm{~cm}$, as shown in Fig. 1(b). This robot measures the local light intensity $I_{t}=I\left(x_{t}, y_{t}\right)$ corresponding to its position $\left(x_{t}, y_{t}\right)$ at time $t$ using eight infrared sensors evenly distributed around its circumference, and adjusts its propulsion speed $v(I)$ accordingly, while randomly changing its orientation with a characteristic reorientation time $\tau=1 \mathrm{~s}$. Its motion can be described by modifying Eq. (1) as

$$
\left\{\begin{array}{l}
\frac{d x_{t}}{d t}=v\left(I_{t}\right) \cos \phi_{t}, \\
\frac{d y_{t}}{d t}=v\left(I_{t}\right) \sin \phi_{t}, \\
\frac{d \phi_{t}}{d t}=\sqrt{\frac{2}{\tau}} \eta_{t} .
\end{array}\right.
$$

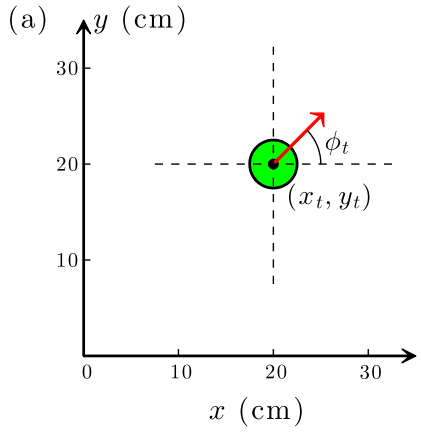

(b)

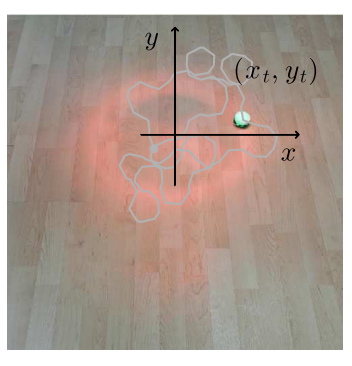

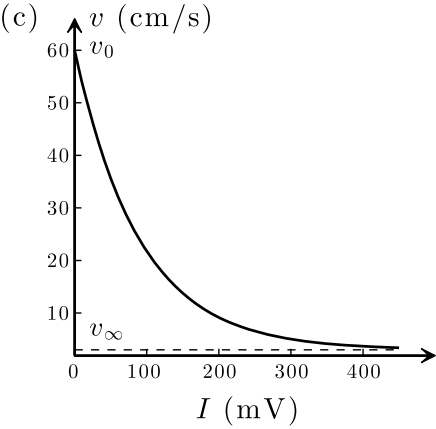

FIG. 1. (a) An autonomous agent, whose position at time $t$ is $\left(x_{t}, y_{t}\right)$, moves with speed $v$ in the direction described by $\phi_{t}$, corresponding to its instantaneous orientation (arrow), which varies randomly with a characteristic time $\tau$. (b) Picture of a phototactic robot in a light gradient generated by an infrared lamp. The propulsion speed of the robot depends on the instantaneously measured light intensity, while its orientation changes randomly. A sample trajectory is shown by the gray solid line. (c) Relation between the measured light intensity $I$ and the robot's speed $v$ [Eq. (3)]. 
Figure 1(b) also shows a sample trajectory (gray solid line) superimposed onto the picture of the robot. The function $v(I)$ is plotted in Fig. 1(c); its functional form is

$$
v(I)=\left(v_{0}-v_{\infty}\right) e^{-I / I_{\mathrm{c}}}+v_{\infty},
$$

where $v_{0}=60 \mathrm{~cm} \mathrm{~s}^{-1}$ is the maximum speed (corresponding to a null intensity), $I_{\mathrm{c}}=90 \mathrm{mV}$ is the characteristic intensity scale (measured in volts) over which the velocity decays, and $v_{\infty}=3 \mathrm{~cm} \mathrm{~s}^{-1}$ is the residual velocity (in the limit of infinite light intensity). It can be seen in Fig. 1(b) that the runs between consecutive turns are longer in the low-intensity (high-speed) regions, while they are shorter in the high-intensity (low-speed) regions. The result is that over a long period of time, the robot spends more time in the high-intensity regions. As we will see, this behavior is in agreement with our theoretical results given in Eq. (7). This is also in agreement with the behavior of chemotactic cells whose explorative behavior decreases when they reach regions with ideal conditions and reduce their locomotion activity in favor of other metabolic activities [2].

We now proceed to add a delay $\delta$ in the agent's response to the measured intensity, which is the main novelty of our work. With this addition, the equations describing the motion of the robot become

$$
\left\{\begin{array}{l}
\frac{d x_{t}}{d t}=v\left(I_{t-\delta}\right) \cos \phi_{t}, \\
\frac{d y_{t}}{d t}=v\left(I_{t-\delta}\right) \sin \phi_{t}, \\
\frac{d \phi_{t}}{d t}=\sqrt{\frac{2}{\tau}} \eta_{t} .
\end{array}\right.
$$

The idea of introducing a sensorial delay is inspired by the way in which bacteria react to a chemotactic gradient; in fact, chemotactic bacteria make a comparison of the number of molecules they detect around themselves at consecutive times in order to decide how to adapt their motion $[2,14,25]$. The presence of sensorial delays is typically ignored, or treated as a nuisance to be controlled [26], while only few theoretical works have considered its possible constructive effects but in situations different from the one studied in this work [27,28]. By introducing a delay long enough so that the robot has enough time to randomize its direction of motion before responding to the sensorial input by changing its speed, we can observe that the motion becomes more directed towards the highintensity (low-speed) region, as can be observed by comparing the trajectories in Fig. 2(a) (with delay $\delta=+5 \tau)$ to that in Fig. 1(b) (without delay).

The system's behavior becomes even more interesting if a "negative" delay is introduced, i.e., if a prediction of the future measured intensity is employed to determine the current robot speed. While it is straightforward to see how a positive delay is introduced (e.g., by a delay in the (a)
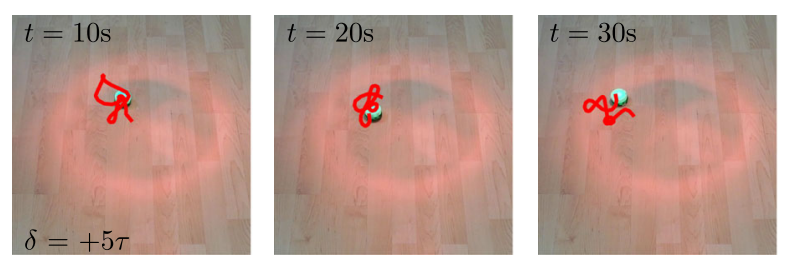

(b)
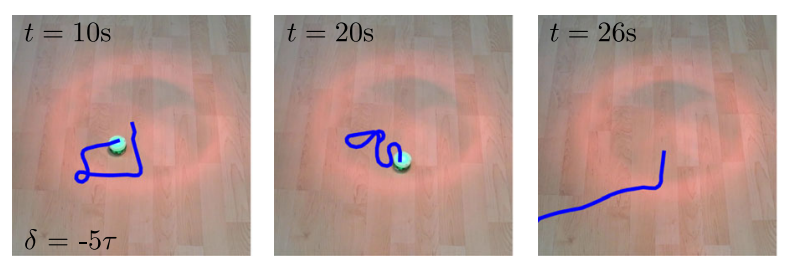

(c)

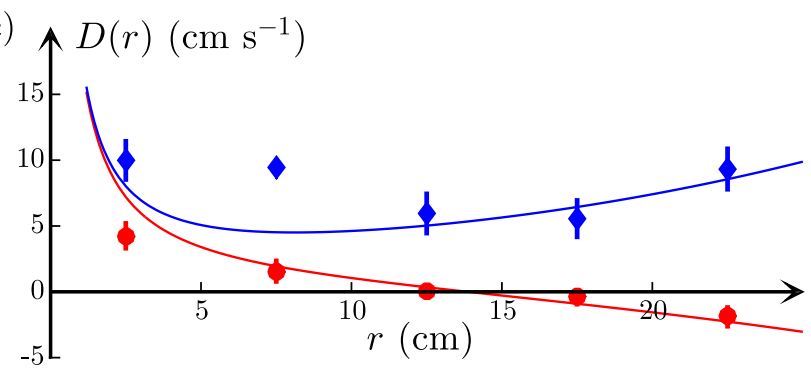

(d)

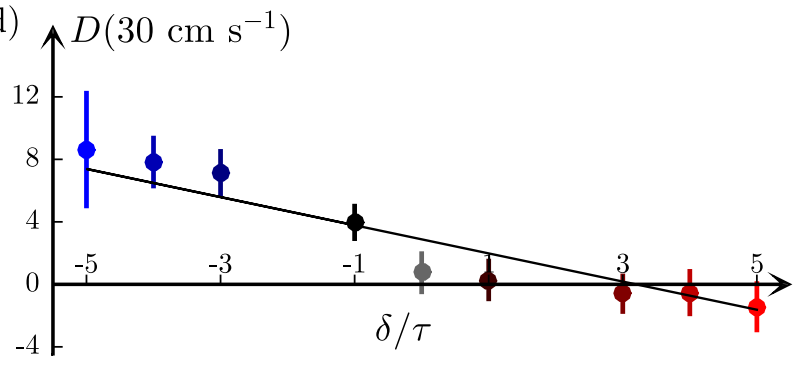

FIG. 2. The long-term behavior of a robot in the light gradient generated by an infrared lamp changes depending on the delay with which it adjusts its speed in response to the sensorial input, i.e., the measured light intensity. The sensorial delay was introduced by linearizing the measured light intensity as a function of time and by extrapolating its past or future value. (a) For positive delays $(\delta=+5 \tau)$, the tendency of the robot to move towards the highintensity (low-speed) regions is enhanced, when compared to the case without delay presented in Fig. 1(b). (b) For negative delays $(\delta=-5 \tau)$, the robot tends to move towards the low-intensity (highspeed) regions. In both cases, the trajectories are shown for a period of $10 \mathrm{~s}$ preceding the time indicated on the plot, and the robot is shown at the final position. (c) Radial drift $D(r)$ calculated according to Eq. (5) from a 40-minute trajectory for the cases of positive (circles) and negative (diamonds) delays. (d) Radial drift calculated according to Eq. (5) when the robots are at $30 \mathrm{~cm}$ from the center of the illuminated area as a function of $\delta / \tau$. The solid lines in (c) and (d) correspond to the theoretically predicted radial drifts given by Eq. (6).

transmission of the signal or by a lapse time before reacting to the signal), the introduction of a negative delay is less intuitive. In fact, a negative delay can be rationalized as a prediction of the future state of the system, which can be 
done based on the signal received up to the present time. For example, in the case of our robots, a negative delay is introduced by linearizing the light intensity measurement as a function of time and extrapolating it into the future, i.e., $I(t-\delta) \approx I(t)-\delta I^{\prime}(t)$, where both $I(t)$ and $I^{\prime}(t)$ are known at time $t$; higher-order predictor algorithms are also possible, making use of more information about the evolution of the intensity measured up to the present. We show the corresponding trajectory in Fig. 2(b), where $\delta=-5 \tau$. In this case, the robot escapes from the highintensity region and moves towards the edge, where the infrared lamp intensity is lower (and the speed higher).

In order to quantify these observations, we have measured the effective radial drift of the robots, which is calculated [29] as

$$
D(r)=\frac{1}{\Delta t}\left\langle r_{n+1}-r_{n} \mid r_{n} \cong r\right\rangle,
$$

where $r$ is the radial coordinate, $r_{n}$ are samples of the robot's radial position, and $\Delta t$ is the time step between samples. The results are shown in Figs. 2(c) and 2(d). For positive delay (red circles), the negative drift for large radial distance shows that the robot tends to move towards the central high-intensity region. For negative delay (blue diamonds), the positive drift shows that the robot escapes from the central high-intensity region. We have also theoretically calculated the radial drift for an autonomous agent whose motion is governed by Eq. (4) (the derivation

(a)
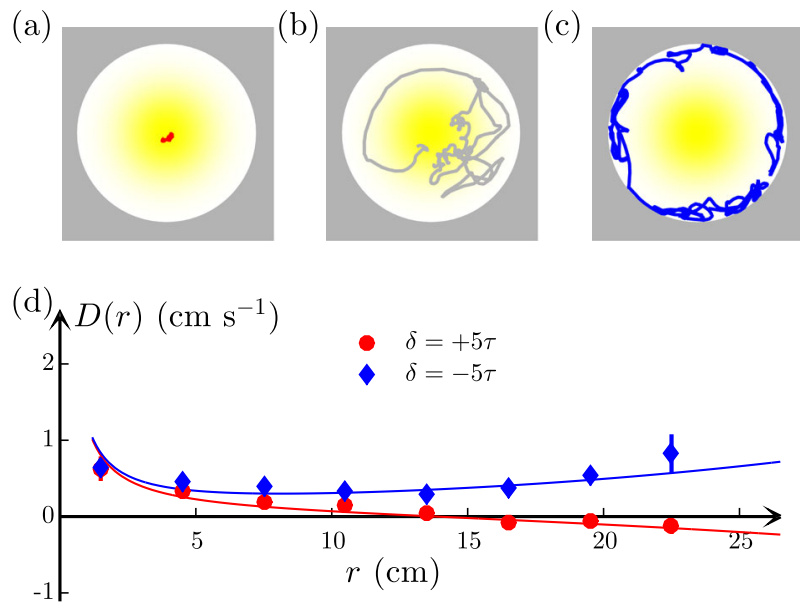

FIG. 3. Simulated trajectories for autonomous agents in an intensity field (background yellow shading) for delays (a) $\delta=+5 \tau$, (b) $\delta=0$, and (c) $\delta=-5 \tau$. The agents are confined in a circular well (gray border). (d) Radial drift as a function of the delay calculated from 1000 simulated trajectories of the system evolving for $100 \mathrm{~s}$ for the cases of positive (circles) and negative (diamonds) delays. The solid lines correspond to the theoretically predicted radial drift given by Eq. (6). Comparing these results with the ones shown in Fig. 2, we observe a good agreement between simulation and experiment. is outlined in Appendix A and described in detail in Appendix B), obtaining

$$
D(r)=\frac{\tau}{2}\left(1-\frac{\delta}{\tau}\right) v(r) \frac{d v}{d r}(r)+\frac{\tau v(r)^{2}}{r}
$$

where $v(r)=v(I(r))$ and we have assumed a radially symmetric intensity distribution. The solid lines plotted in Figs. 2(c) and 2(d) show that there is a good agreement between these theoretical predictions and the experimentally measured data. We have further corroborated these results with numerical simulations, whose results, shown in Fig. 3, are in good agreement with the experimental results shown in Fig. 2. The numerical simulations were performed by solving the finite difference approximation of Eq. (4) $[20,30]$. The delayed sensorial measurement was evaluated by the Taylor expansion of the measured intensity about the agent's location and extrapolating the corresponding past or future value.
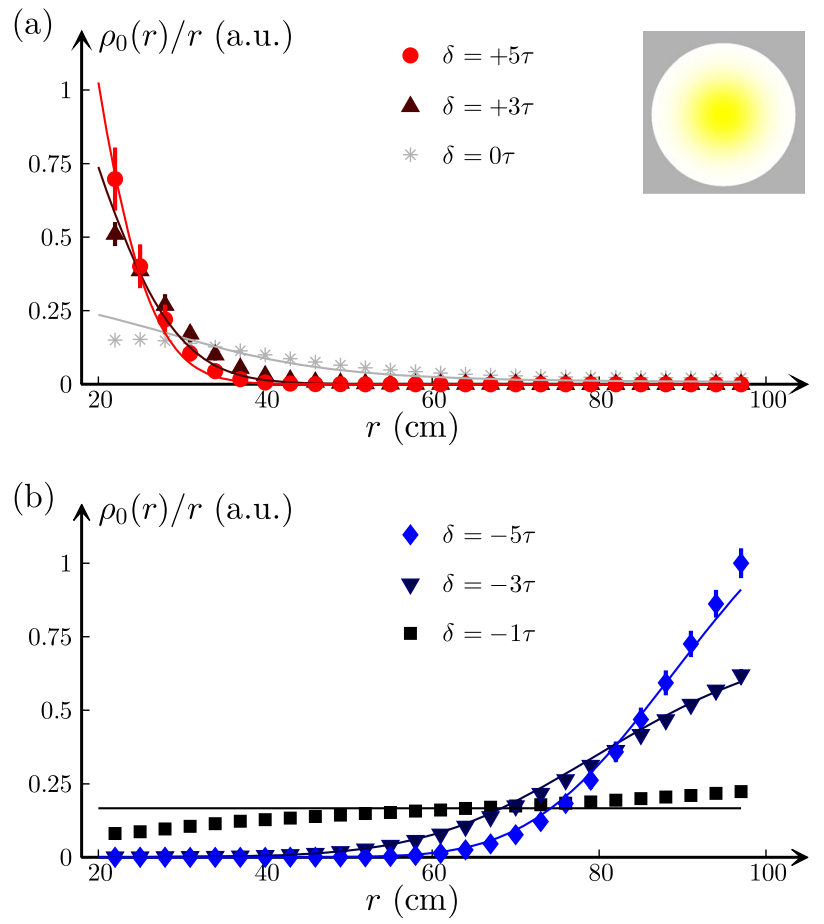

FIG. 4. Theoretically predicted radial probability distribution of the position of an agent [Eq. (7)] moving in a radial intensity field [in the inset in (a), the agent is confined in a circular well with radius $100 \mathrm{~cm}$ indicated by the gray border] as a function of the sensorial delay time: (a) for $\delta>-\tau$, the agent tends to spend more time in the low-speed (high-intensity) central region; (b) for $\delta<-\tau$, the agent spends more time in the high-speed (lowintensity) peripheral region; for $\delta=-\tau$, the probability distribution is uniform (black line). These results are corroborated by numerical simulations of autonomous agents shown by the symbols. For each case, we have simulated a very long trajectory $\left(10^{8} \mathrm{~s}\right)$ to obtain an accurate and smooth distribution. 
We can also theoretically derive the approximate steadystate probability distribution of the agent's position (the derivation is outlined in Appendix A and described in detail in Appendix B), which exists and equals

$$
\rho_{0}(x, y)=\frac{1}{N v(x, y)^{1+(\delta / \tau)}},
$$

provided that the normalization constant

$$
N=\int v(x, y)^{-[1+(\delta / \tau)]} d x d y<\infty
$$

Equation (6) confirms our initial observations that the larger the positive delay is [solid lines in Fig. 4(a)], the more time the agent spends in the low-speed (highintensity) regions. On the other hand, the more negative the delay is [solid lines in Fig. 4(b)], the more time the agent spends in the high-speed (low-intensity) regions. Interestingly, we note that there is a cutoff value at $\delta=-\tau$ for which the probability distribution of the agent is uniform [black solid line in Fig. 4(b)]. We have further corroborated these results with numerical simulations shown by the symbols in Figs. 4(a) and 4(b).

We emphasize that the qualitative change of the particle's behavior occurs at a negative delay, i.e., $\delta=-\tau$. Introduction of negative delays is thus crucial for the described transition. On the other hand, positive delays also strongly influence the system's behavior. While without delay the particle spends more time in slow regions, a positive delay makes this tendency more pronounced, as clearly seen at the quantitative level from Eq. (7). This tendency persists, albeit in a weaker form, for small negative delays $-\tau<\delta<0$ and gets reversed at the critical value $\delta=-\tau$.

\section{MULTIPLE AGENTS}

We can now build on these observations to engineer the large-scale organization of groups of robots. In order to do this, each robot must be able not only to sense the local intensity, but also to create a luminosity field. Thus, we have equipped each robot with six LEDs evenly placed around its circumference (EDEI-1LS3) [as shown in Fig. 5(a)], which emit infrared light (wavelength $850 \mathrm{~nm}$ ) so that each robot generates a decaying light intensity around itself. The LEDs are arranged so that the robot measures only the light intensity emitted by the other robots. A phototactic robot capable of measuring this light intensity will be able to move in the resulting field similarly to the case discussed above, i.e., that of the light intensity generated by a static infrared lamp. We stress that each robot only measures the local intensity without being aware of the positions of the other robots.

We have experimentally studied how three autonomous robots organize by reacting to the cumulative light field (a)

(b)

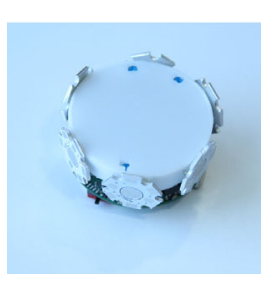

(d)

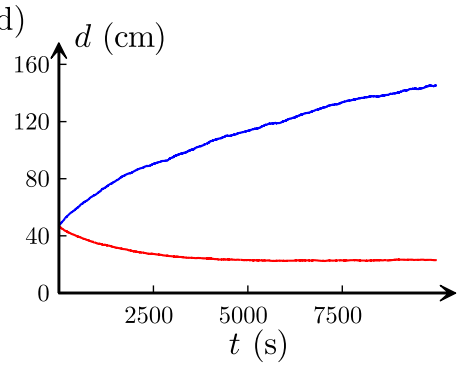

$t=25 s$

$t=50 \mathrm{~s}$

$t=90 s$

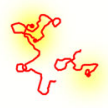

8

$\delta=+3 \tau$

(c)

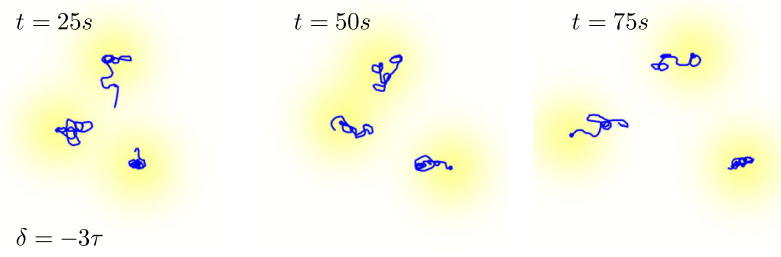

FIG. 5. (a) Picture of a phototactic robot equipped with six infrared LEDs so that it can emit a radially decaying light intensity around itself. (b) A group of three such robots, which adjust their speed as a function of the sensed light intensity, aggregate and form a dynamic cluster if their sensorial delay is positive $(\delta=+3 \tau)$ and (c) segregate if it is negative $(\delta=-3 \tau)$. In each panel in (b) and (c), the trajectories are shown for a period of $10 \mathrm{~s}$ preceding the time indicated on the plot, and the dot indicates the final position of the robot. (d) Average distance $d$ between agents in a group of three simulated autonomous agents as a function of time: For positive delays, as the agents tend to come together and form a cluster, their average distance decreases over time; for negative delays, as the agents move apart and explore a larger area, their average distance increases.

created by all of them as a function of their sensorial delay. For a positive sensorial delay $[\delta=+3 \tau$, Fig. 5(b)], the three robots gradually move towards each other and form a dynamic cluster, which remains stable over time. A single robot's tendency to spend more time in the high-intensity regions when there is positive delay leads to multiple robots forming clusters because of their preference for high-intensity regions. For a negative delay $[\delta=-3 \tau$, Fig. 5(c)], the three robots tend to move away from each other, dispersing and exploring a much larger area. In order to understand this behavior in a more quantitative way, we have also simulated a larger number of trajectories for a group of three agents and plotted the average distance between the agents as a function of time for various sensorial delays. The results are reported in Fig. 5(d): For positive delays, as the agents tend to come together and form a cluster, their average distance decreases over time; for negative delays, as the agents move apart and explore a 


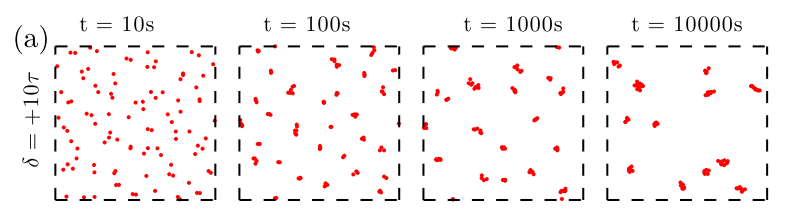

(b)
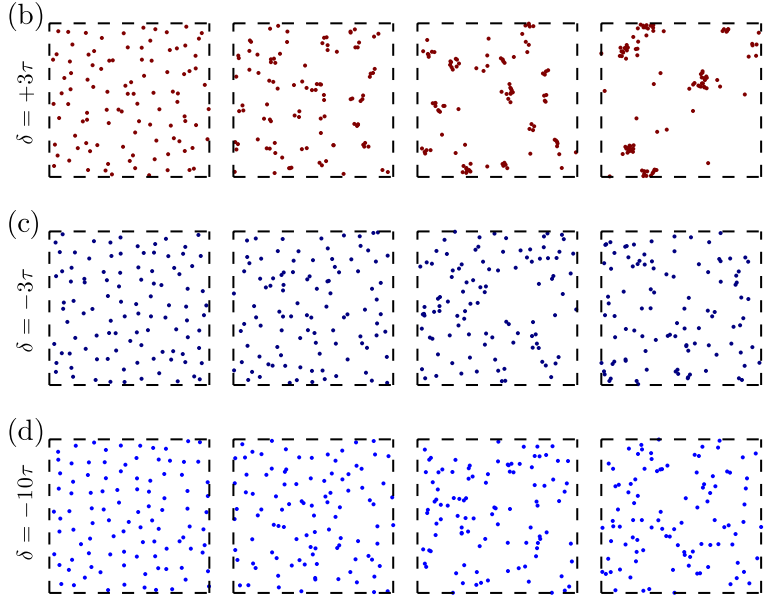

FIG. 6. Simulation of the long-term behavior of an ensemble of 100 autonomous agents that emit a radially decaying intensity field and adjust their speed depending on the measured local intensity. Depending on the sensorial delay, the long-term behavior and large-scale organization are significantly different. $(a, b)$ In the case of positive delays, the agents come together and form metastable clusters. (c,d) In the case of negative delays, they explore the space, staying away from each other.
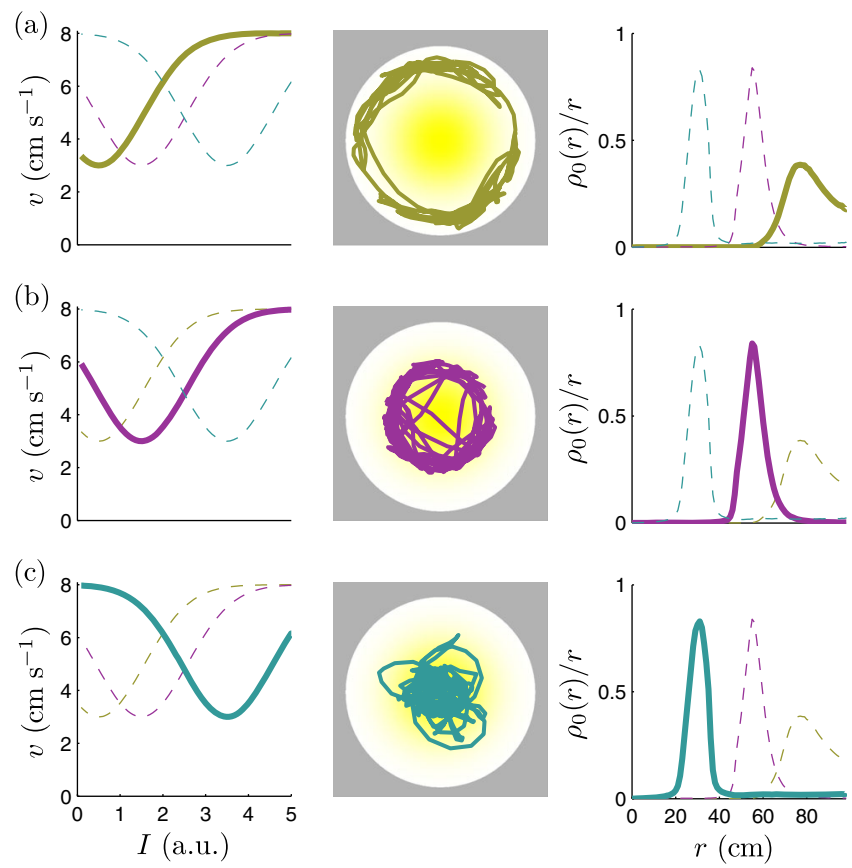

FIG. 7. The long-term behavior of autonomous agents can be tuned by changing the function that relates the speed to the measured intensity. For a fixed sensorial delay $(\delta=+10 \tau)$, we consider various speed-intensity relations (first column), which lead to different particle behaviors (center column) and different stationary probability densities (last column). larger area, their average distance increases. The qualitative change of the agents' behavior occurs at a strictly negative value of the dimensionless parameter $\delta / \tau=-1$ [see Eq. (7)]. While introduction of negative delays is thus crucial for the described transition from aggregation to segregation, positive delays also strongly influence the system's behavior by enhancing the tendency of the agents to aggregate. Importantly, not only a light field, but any radially decaying scalar (e.g., chemical, acoustic) field created by the autonomous agents can be used in order to achieve this kind of control over their behavior.

In order to explore the scalability of this mechanism, we have simulated the behavior of an ensemble of 100 robots. Each robot emits a field that decays radially like a Gaussian and responds to the locally measured cumulative intensity by adjusting its speed. The long-term behavior and the large-scale organization of these ensembles of agents significantly depend on the sensorial delay, as shown in Fig. 6. For positive delay, they move collectively by forming clusters [Figs. 6(a) and 6(b)]. On the other hand, for negative delays, they move away from each other in order to reduce the intensity that each of them measures and are thus able to explore the space more effectively [Figs. 6(c) and 6(d)]. The possibility of tuning the sensorial

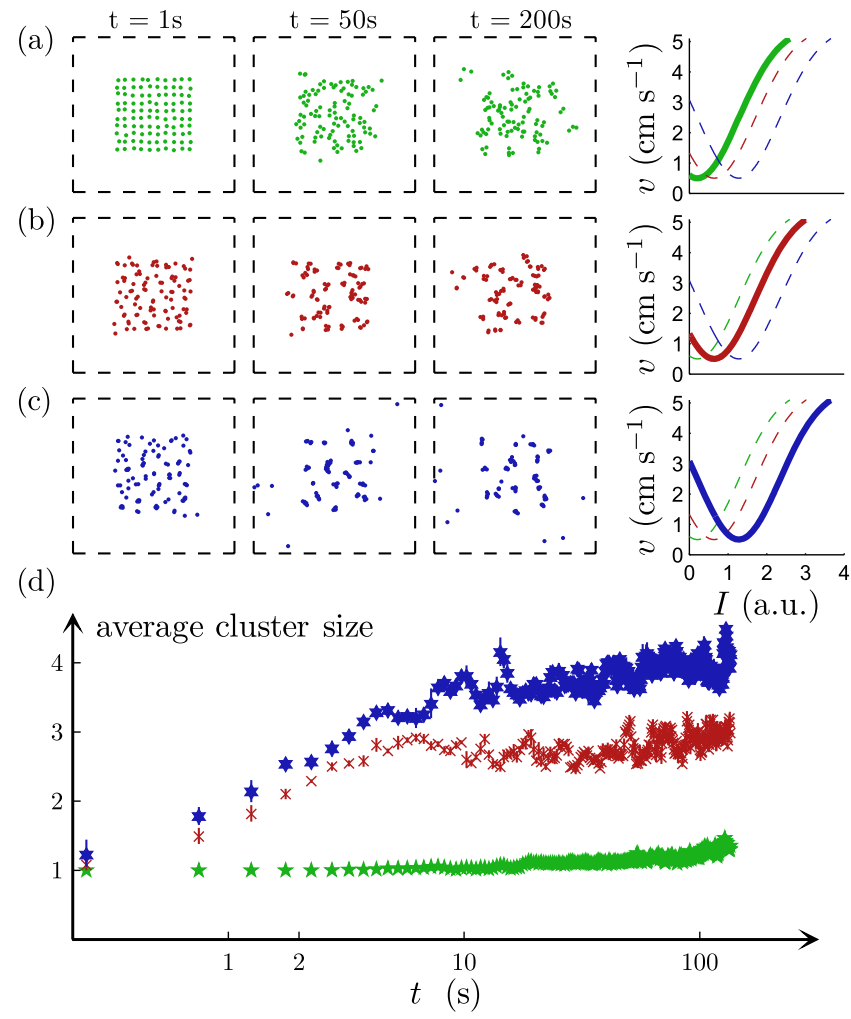

FIG. 8. (a)-(c) 100 agents whose speed-intensity relation (last column) is nonautonomous form dynamic clusters whose (d) characteristic average size depends on the intensity corresponding to the minimum speed: The higher the intensity, the larger the average cluster size. 
delay can be exploited, for example, in a search-and-rescue task by initially setting a negative delay so that the robots can thoroughly explore the environment and, at a later stage, a positive delay so that the robots can be collected into clusters to share the gathered information. Collecting all robots can also be easily achieved by sending a strong signal capable of eclipsing the signals emitted by the robots themselves.

It is also possible to adjust the behavior of the agents by altering the intensity-speed relation to something different than Eq. (3). For example, instead of a monotonically decreasing relation, it is possible to use a relation with a minimum at some specific value. As can be seen in Fig. 7, this alters the agent's behavior so that it spends more time where the intensity corresponds to the minimum speed. In this way, it is possible to control where the agent will spend most of its time, which may be useful, e.g., for targeted delivery. Furthermore, in the presence of multiple agents capable of emitting a radially decaying intensity field, changing the intensity-speed relation permits one to control various features of the clusters such as their characteristic size, as shown in Fig. 8.

\section{SINGLE AND MULTIPLE ROBOTS IN THREE DIMENSIONS}

Our results can also be extended to the three-dimensional case, where they still hold with only minor adjustments. This could be important when considering airborne objects (e.g., drones, flying insects, birds) or underwater objects (e.g., fish, submarine robots). In three dimensions, the autonomous agent motion can be modeled by the set of equations

$$
\left\{\begin{array}{l}
\frac{d x_{t}}{d t}=v\left(I_{t-\delta}\right) \sin \theta_{t} \cos \phi_{t}, \\
\frac{d y_{t}}{d t}=v\left(I_{t-\delta}\right) \sin \theta_{t} \sin \phi_{t}, \\
\frac{d z_{t}}{d t}=v\left(I_{t-\delta}\right) \cos \theta_{t}, \\
\frac{d \theta_{t}}{d t}=\frac{1}{\tau} \cot \theta_{t}+\sqrt{\frac{2}{\tau}} \eta_{t}^{(1)}, \\
\frac{d \phi_{t}}{d t}=\frac{1}{\sin \theta_{t}} \sqrt{\frac{2}{\tau}} \eta_{t}^{(2)},
\end{array}\right.
$$

where $\left(x_{t}, y_{t}, z_{t}\right)$ is the position of the agent at time $t, \theta_{t}$ and $\phi_{t}$ are its azimuthal and polar orientations, respectively, and $\eta_{t}^{(1)}$ and $\eta_{t}^{(2)}$ are independent white noises. Similar equations but without delay have already been considered, e.g., in Ref. [31], to describe active Brownian motion in three dimensions. The last two equations describe (accelerated) Brownian motion on the surface of the unit sphere. From this model, we obtain the approximate steady-state probability distribution (the derivation is described in Appendix B), which exists and equals

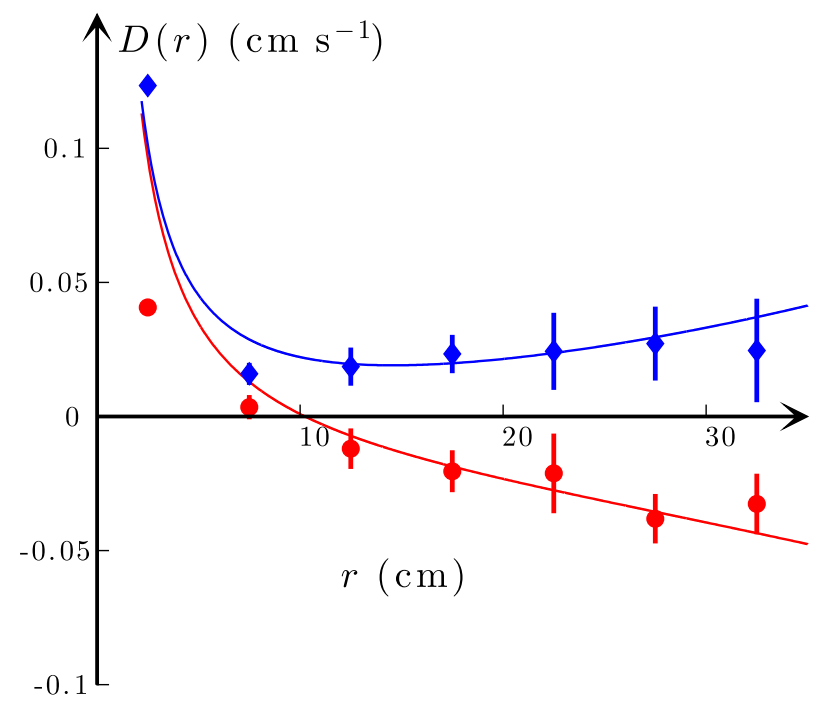

FIG. 9. Radial drift for an agent moving in a three-dimensional radially decaying intensity field. The symbols represent the results of numerical simulations, and the solid lines show the corresponding theoretically predicted values [see Eq. (B21)]. For positive delays (red circles and line), there is a negative radial drift that pushes the agent towards the central high-intensity (low-speed) region. For negative delays (blue diamonds and line), there is a positive radial drift that pushes the agent away from the high-intensity region and towards the peripheral low-intensity (high-speed) region.
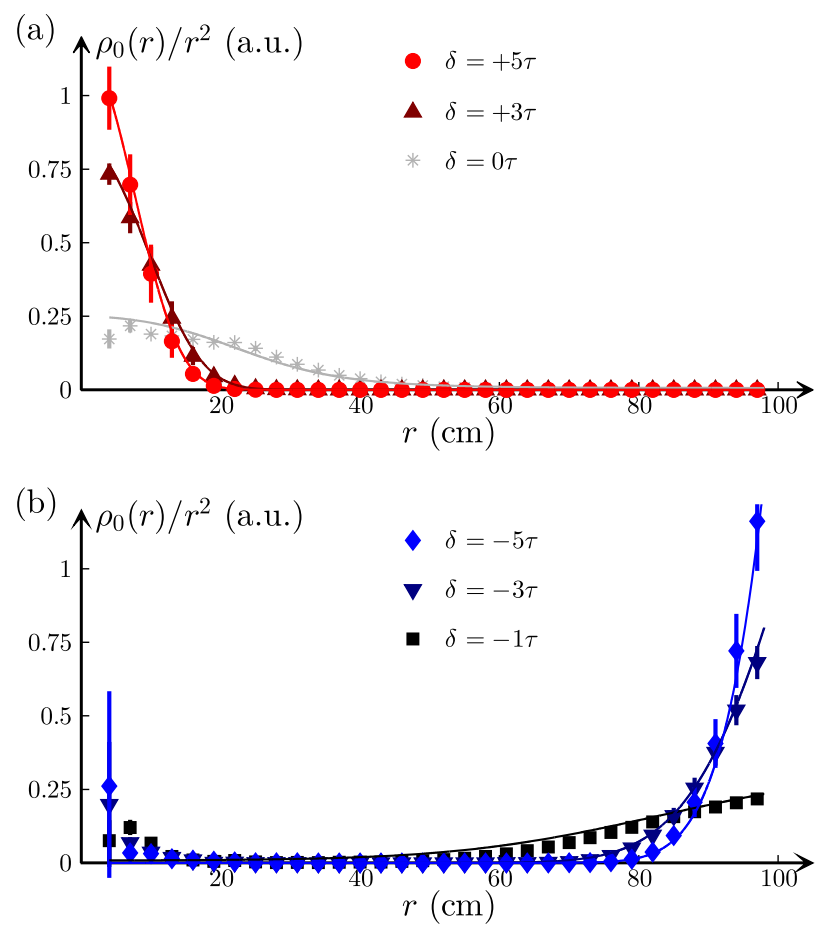

FIG. 10. Probability distribution in the radial direction for a single agent moving in a three-dimensional spherical well (radius $100 \mathrm{~cm}$ ) with a radially decaying intensity field. The probability densities obtained from simulations are denoted by the symbols, and the corresponding theoretical predictions [Eq. (9)] are shown by the solid lines. 


$$
\rho_{0}(x, y, z)=\frac{1}{M v(x, y, z)^{1+2(\delta / \tau)}}
$$

provided that the normalization constant

$$
M=\int v(x, y, z)^{-[1+2(\delta / \tau)]} d x d y d z<\infty
$$

Comparing Eq. (9) and Eq. (7), we note that the main difference is that in the three-dimensional case, the uniform distribution occurs for $\delta=-0.5 \tau$ instead of for $\delta=-\tau$. Otherwise, the agents still exhibit a qualitatively different behavior for positive and negative sensorial delay, corresponding, respectively, to an effective drift towards highintensity and low-intensity regions, as illustrated in Figs. 9 and 10. As in the two-dimensional case, in the threedimensional case, it is also possible to engineer this drift by changing the time delay in order to tune the collective behavior of a swarm from aggregation and clustering to segregation.

\section{CONCLUSION}

We have demonstrated the use of delayed sensorial feedback to control the organization of an ensemble of autonomous agents. We realized this model experimentally by using autonomous robots, further backed it up with simulations, and finally provided a mathematical analysis that agrees with the results obtained in the experiments and simulations. Our findings show that a single robot, measuring the intensity locally, spends more time in either a high- or a low-intensity region depending on its sensorial delay. Tuning the value of the delay permits one to engineer the behavior of an ensemble of robots so that they come together or separate from each other. The robustness and flexibility of these behaviors are very promising for applications in the field of swarm robotics $[6,10,11,16,18]$, as well as in the assembly of nanorobots, e.g., for targeted delivery within tissues. Furthermore, since some living entities, such as bacteria, are known to respond to temporal evolution of stimuli [2,25], the presence of a sensorial delay could also explain the swarming behavior of groups of living organisms.

\section{ACKNOWLEDGMENTS}

The authors thank Gilles Caprari (GCtronic) for his help with the robots. G. V. thanks Holger Stark for useful discussions that led to the original idea for this work. J. W. and A. M. were partially supported by the NSF Grants No. DMS 1009508 and No. DMS 0623941. The work of J. W. was partially supported by NSF under Grant No. DMS 1440140 while he was in residence at the Mathematical Sciences Research Institute in Berkeley during 2015. G. V. was partially supported by Marie Curie Career Integration Grant (MC-CIG) No. PCIG11 GA-2012-321726 and the Turkish Academy of Sciences
(TÜBA). M. M. was partially supported by Tubitak Grant No. $113 Z 556$.

\section{APPENDIX A: MATHEMATICAL DERIVATION-AN OUTLINE}

We studied the limit of the system (4) as $\delta, \tau \rightarrow 0$ at the same rate so that $\delta=c \epsilon$ and $\tau=k \epsilon$, where $c$ and $k$ remain constant in the limit $\delta, \tau, \epsilon \rightarrow 0$. We expanded $v$ about $t$ to first order in $\delta$ and solved the resulting equations for $\dot{x}$ and $\dot{y}$. We expanded the resulting system to first order in the small parameter $\delta / \sqrt{\tau}$. We then considered the corresponding backward Kolmogorov equation for the probability density $\rho$. We expanded $\rho$ in powers of the parameter $\sqrt{\epsilon}$, i.e., $\rho=\rho_{0}+\sqrt{\epsilon} \rho_{1}+\epsilon \rho_{2}+\cdots$, and used the standard multiscale expansion method [32] to derive the backward Kolmogorov equation for the limiting density $\rho_{0}$ :

$$
\frac{\partial \rho_{0}}{\partial t}=\frac{\tau}{2}\left(1-\frac{\delta}{\tau}\right) v\left(\frac{\partial v}{\partial x} \frac{\partial \rho_{0}}{\partial x}+\frac{\partial v}{\partial y} \frac{\partial \rho_{0}}{\partial y}\right)+\frac{\tau v^{2}}{2} \Delta \rho_{0} .
$$

From this equation, we got the limiting SDE:

$$
\left\{\begin{array}{l}
d x_{t}=\frac{\tau}{2}\left(1-\frac{\delta}{\tau}\right) v\left(x_{t}, y_{t}\right) \frac{\partial v}{\partial x}\left(x_{t}, y_{t}\right) d t+\sqrt{\tau} v\left(x_{t}, y_{t}\right) d W_{t}^{1}, \\
d y_{t}=\frac{\tau}{2}\left(1-\frac{\delta}{\tau}\right) v\left(x_{t}, y_{t}\right) \frac{\partial v}{\partial y}\left(x_{t}, y_{t}\right) d t+\sqrt{\tau} v\left(x_{t}, y_{t}\right) d W_{t}^{2},
\end{array}\right.
$$

where $W^{1}$ and $W^{2}$ are independent Wiener processes. Assuming that $v$ is rotationally invariant, from Eq. (A2), we obtain the formula for the radial drift [Eq. (6)]:

$$
D(r)=\frac{\tau}{2}\left(1-\frac{\delta}{\tau}\right) v(r) \frac{d v}{d r}(r)+\frac{\tau v(r)^{2}}{r} .
$$

Setting the right-hand side of the forward (Fokker-Planck) equation corresponding to Eq. (A1) equal to zero, we get the formula for the stationary probability density $\rho_{0}$ (if it exists) [Eq. (7)],

$$
\rho_{0}(x, y)=\frac{1}{N v(x, y)^{1+(\delta / \tau)}},
$$

where $N$ is the normalization constant. A similar analysis follows for the three-dimensional case, leading to the threedimensional stationary probability density given by Eq. (9). A more detailed derivation is provided in the following appendix.

\section{APPENDIX B: MATHEMATICAL DERIVATION-DETAILS}

\section{Mathematical derivation in two dimensions}

Our motivation is the system given by Eq. (4), which we can rewrite as 


$$
\left\{\begin{array}{l}
\frac{d x_{t}}{d t}=v\left(x_{t-\delta}, y_{t-\delta}\right) \cos \phi_{t} \\
\frac{d y_{t}}{d t}=v\left(x_{t-\delta}, y_{t-\delta}\right) \sin \phi_{t} \\
\frac{d \phi_{t}}{d t}=\sqrt{\frac{2}{\tau}} \eta_{t}
\end{array}\right.
$$

While the reorientation characteristic time $\tau$ is constant in the experiment, to analyze the system mathematically we study the limit as $\tau$ and $\delta$ go to zero. Thus, in the mathematical analysis, we use $\tilde{\tau}$ and $\tilde{\delta}$ to represent the reorientation characteristic time and time delay, in order to differentiate these parameters that will go to zero from the reorientation characteristic time $\tau$ and time delay $\delta$ in the experiment. If $\tau$ is very small, a particle that moves according to the above equations changes direction very rapidly; thus, for the displacement of this particle from its initial position to be significant, the particle must have a large speed. We account for this mathematically by letting $v$ increase as $\tilde{\tau}$ decreases. In order to obtain nontrivial behavior in the limit, we must define $v=(u / \sqrt{\tilde{\tau}})$, where $u$ does not depend on $\tilde{\tau}$. Then, we have

$$
\left\{\begin{aligned}
d x_{t} & =\frac{1}{\sqrt{\tilde{\tau}}} u\left(x_{t-\tilde{\delta}}, y_{t-\tilde{\delta}}\right) \cos \phi_{t} d t, \\
d y_{t} & =\frac{1}{\sqrt{\tilde{\tau}}} u\left(x_{t-\tilde{\delta}}, y_{t-\tilde{\delta}}\right) \sin \phi_{t} d t, \\
d \phi_{t} & =\sqrt{\frac{2}{\tilde{\tau}}} d W_{t},
\end{aligned}\right.
$$

where $W_{t}$ is a Wiener process.

We expand $u$ about $t$ to first order in $\tilde{\delta}$. The resulting system approximates the above equations and is also the system actually used in numerical simulation. We thus study the approximate equations:

$$
\left\{\begin{array}{l}
\dot{x}_{t}=\frac{1}{\sqrt{\tilde{\tau}}}\left[u\left(x_{t}, y_{t}\right)-\tilde{\delta} \frac{\partial u}{\partial x}\left(x_{t}, y_{t}\right) \dot{x}_{t}-\tilde{\delta} \frac{\partial u}{\partial y}\left(x_{t}, y_{t}\right) \dot{y}_{t}\right] \cos \phi_{t} \\
\dot{y}_{t}=\frac{1}{\sqrt{\tilde{\tau}}}\left[u\left(x_{t}, y_{t}\right)-\tilde{\delta} \frac{\partial u}{\partial x}\left(x_{t}, y_{t}\right) \dot{x}_{t}-\tilde{\delta} \frac{\partial u}{\partial y}\left(x_{t}, y_{t}\right) \dot{y}_{t}\right] \sin \phi_{t} \\
\phi_{t}=\sqrt{\frac{2}{\tilde{\tau}}} W_{t}
\end{array}\right.
$$

Solving the first two equations for $\dot{x}_{t}$ and $\dot{y}_{t}$, we get

$$
\left\{\begin{array}{l}
\dot{x}_{t}=\frac{1}{\sqrt{\tau}} u\left(x_{t}, y_{t}\right) \cos \phi_{t} \times\left[1+\frac{\tilde{\delta}}{\sqrt{\tilde{\tau}}}\left(\frac{\partial u}{\partial x}\left(x_{t}, y_{t}\right) \cos \phi_{t}+\frac{\partial u}{\partial y}\left(x_{t}, y_{t}\right) \sin \phi_{t}\right)\right]^{-1} \\
\dot{y}_{t}=\frac{1}{\sqrt{\tau}} u\left(x_{t}, y_{t}\right) \sin \phi_{t} \times\left[1+\frac{\tilde{\delta}}{\sqrt{\tilde{\tau}}}\left(\frac{\partial u}{\partial x}\left(x_{t}, y_{t}\right) \cos \phi_{t}+\frac{\partial u}{\partial y}\left(x_{t}, y_{t}\right) \sin \phi_{t}\right)\right]^{-1} \\
\phi_{t}=\sqrt{\frac{2}{\tilde{\tau}}} W_{t} .
\end{array}\right.
$$

We approximate the system further, for $(\tilde{\delta} / \sqrt{\tilde{\tau}}) \ll 1$, by

$$
\left\{\begin{array}{l}
d x_{t}=\frac{1}{\sqrt{\tilde{\tau}}} u\left(x_{t}, y_{t}\right) \cos \phi_{t} \times\left[1-\frac{\tilde{\delta}}{\sqrt{\tilde{\tau}}}\left(\frac{\partial u}{\partial x}\left(x_{t}, y_{t}\right) \cos \phi_{t}+\frac{\partial u}{\partial y}\left(x_{t}, y_{t}\right) \sin \phi_{t}\right)\right] d t \\
d y_{t}=\frac{1}{\sqrt{\tilde{\tau}}} u\left(x_{t}, y_{t}\right) \sin \phi_{t} \times\left[1-\frac{\tilde{\delta}}{\sqrt{\tilde{\tau}}}\left(\frac{\partial u}{\partial x}\left(x_{t}, y_{t}\right) \cos \phi_{t}+\frac{\partial u}{\partial y}\left(x_{t}, y_{t}\right) \sin \phi_{t}\right)\right] d t \\
d \phi_{t}=\sqrt{\frac{2}{\tilde{\tau}}} d W_{t} .
\end{array}\right.
$$

We study the limit of the system (B1) as $\tilde{\delta}$ and $\tilde{\tau}$ go to zero at the same rate. This is consistent with the assumption $(\tilde{\delta} / \sqrt{\tilde{\tau}}) \ll 1$. Thus, we suppose that $\tilde{\delta}$ and $\tilde{\tau}$ stay proportional to a single characteristic time $\epsilon$, i.e., $\tilde{\delta}=c \epsilon$ and $\tilde{\tau}=k \epsilon$, where $c$ and $k$ remain constant in the limit $\tilde{\delta}, \tilde{\tau}, \epsilon \rightarrow 0$. Writing Eq. (B1) in terms of $\epsilon$, we obtain

$$
\left\{\begin{aligned}
d x_{t} & =\left(\frac{1}{\sqrt{k \epsilon}} u \cos \phi_{t}-\frac{c}{k} u \frac{\partial u}{\partial x} \cos ^{2} \phi_{t}-\frac{c}{k} u \frac{\partial u}{\partial y} \cos \phi_{t} \sin \phi_{t}\right) d t \\
d y_{t} & =\left(\frac{1}{\sqrt{k \epsilon}} u \sin \phi_{t}-\frac{c}{k} u \frac{\partial u}{\partial x} \cos \phi_{t} \sin \phi_{t}-\frac{c}{k} u \frac{\partial u}{\partial y} \sin ^{2} \phi_{t}\right) d t \\
d \phi_{t} & =\sqrt{\frac{2}{k \epsilon}} d W_{t}
\end{aligned}\right.
$$

We take the limit $\epsilon \rightarrow 0$ by using the multiscale expansion method (a detailed exposition can be found in, e.g., Ref. [32]). The backward Kolmogorov equation corresponding to the system (B2) of SDEs is 


$$
\begin{aligned}
\frac{\partial \rho}{\partial t}= & \frac{1}{k \epsilon} \frac{\partial^{2} \rho}{\partial \phi^{2}}+\frac{1}{\sqrt{k \epsilon}} u \cos \phi \frac{\partial \rho}{\partial x}+\frac{1}{\sqrt{k \epsilon}} u \sin \phi \frac{\partial \rho}{\partial y} \\
& -\left(\frac{c}{k} u \frac{\partial u}{\partial x} \cos ^{2} \phi+\frac{c}{k} u \frac{\partial u}{\partial y} \cos \phi \sin \phi\right) \frac{\partial \rho}{\partial x} \\
& -\left(\frac{c}{k} u \frac{\partial u}{\partial x} \cos \phi \sin \phi+\frac{c}{k} u \frac{\partial u}{\partial y} \sin ^{2} \phi\right) \frac{\partial \rho}{\partial y}
\end{aligned}
$$

which can be written as

$$
\frac{\partial \rho}{\partial t}=\left(\frac{1}{\epsilon} L_{0}+\frac{1}{\sqrt{\epsilon}} L_{1}+L_{2}\right) \rho,
$$

where

$$
\begin{aligned}
& L_{0}=\frac{1}{k} \frac{\partial^{2}}{\partial \phi^{2}}, \\
& L_{1}=\frac{1}{\sqrt{k}} u \cos \phi \frac{\partial}{\partial x}+\frac{1}{\sqrt{k}} u \sin \phi \frac{\partial}{\partial y},
\end{aligned}
$$

and

$$
\begin{aligned}
L_{2}= & -\left(\frac{c}{k} u \frac{\partial u}{\partial x} \cos ^{2} \phi+\frac{c}{k} u \frac{\partial u}{\partial y} \cos \phi \sin \phi\right) \frac{\partial}{\partial x} \\
& -\left(\frac{c}{k} u \frac{\partial u}{\partial x} \cos \phi \sin \phi+\frac{c}{k} u \frac{\partial u}{\partial y} \sin ^{2} \phi\right) \frac{\partial}{\partial y} .
\end{aligned}
$$

We expand $\rho$ in powers of $\sqrt{\epsilon}$,

$$
\rho=\rho_{0}+\sqrt{\epsilon} \rho_{1}+\epsilon \rho_{2}+\cdots,
$$

and derive the backward Kolmogorov equation for the limiting density $\rho_{0}$. First, substituting Eq. (B4) in Eq. (B3) and equating terms of the same order in $\sqrt{\epsilon}$ gives the equations

$$
O\left(\frac{1}{\epsilon}\right): L_{0} \rho_{0}=\frac{1}{k} \frac{\partial^{2} \rho_{0}}{\partial \phi^{2}}=0
$$

$$
\begin{aligned}
O\left(\frac{1}{\sqrt{\epsilon}}\right): & L_{1} \rho_{0}+L_{0} \rho_{1} \\
= & \frac{1}{\sqrt{k}} u \cos \phi \frac{\partial \rho_{0}}{\partial x}+\frac{1}{\sqrt{k}} u \sin \phi \frac{\partial \rho_{0}}{\partial y}+\frac{1 \partial^{2} \rho_{1}}{k}=0 \\
& O(1): L_{2} \rho_{0}+L_{1} \rho_{1}+L_{0} \rho_{2}=\frac{\partial \rho_{0}}{\partial t} .
\end{aligned}
$$

While $f(x, y, \phi, t)=A(x, y, t) \phi+B(x, y, t)$ is the general solution to Eq. (B5), we expect $\rho_{0}$ to be independent of $\phi$, and therefore, we choose $\rho_{0}=\rho_{0}(x, y, t)$. Next, we find $\rho_{1}$ in terms of $\rho_{0}$ by solving Eq. (B6); since $\rho_{0}$ does not depend on $\phi$, we have

$$
\rho_{1}=\sqrt{k} u \frac{\partial \rho_{0}}{\partial x} \cos \phi+\sqrt{k} u \frac{\partial \rho_{0}}{\partial y} \sin \phi,
$$

where we leave out a linear term in $\phi$ in order to make $\rho_{1}$ periodic in $\phi$. We could include a term that is constant in $\phi$, but this would not change the final result. Equation (B7) implies that the function $\left(\partial \rho_{0} / \partial t\right)-L_{1} \rho_{1}-L_{2} \rho_{0}$ is in the range of the operator $L_{0}$. This is a self-adjoint operator on the space $L^{2}[0,2 \pi]$, so the above function must be orthogonal to its kernel, which, in particular, contains constants. It follows that

$$
\frac{1}{2 \pi} \int_{0}^{2 \pi}\left(\frac{\partial \rho_{0}}{\partial t}-L_{1} \rho_{1}-L_{2} \rho_{0}\right) d \phi=0
$$

so that

$$
\begin{aligned}
\frac{\partial \rho_{0}}{\partial t}= & \frac{1}{2 \pi} \int_{0}^{2 \pi}\left[\left(\frac{1}{\sqrt{k}} u \cos \phi \frac{\partial}{\partial x}+\frac{1}{\sqrt{k}} u \sin \phi \frac{\partial}{\partial y}\right)\right. \\
& \times\left(\sqrt{k} u \frac{\partial \rho_{0}}{\partial x} \cos \phi+\sqrt{k} u \frac{\partial \rho_{0}}{\partial y} \sin \phi\right) \\
& -\left(\frac{c}{k} u \frac{\partial u}{\partial x} \cos ^{2} \phi+\frac{c}{k} u \frac{\partial u}{\partial y} \cos \phi \sin \phi\right) \frac{\partial \rho_{0}}{\partial x} \\
& \left.-\left(\frac{c}{k} u \frac{\partial u}{\partial x} \cos \phi \sin \phi+\frac{c}{k} u \frac{\partial u}{\partial y} \sin ^{2} \phi\right) \frac{\partial \rho_{0}}{\partial y}\right] d \phi,
\end{aligned}
$$

and so

$$
\begin{aligned}
\frac{\partial \rho_{0}}{\partial t}= & \frac{u}{2} \frac{\partial}{\partial x}\left(u \frac{\partial \rho_{0}}{\partial x}\right)+\frac{u}{2} \frac{\partial}{\partial y}\left(u \frac{\partial \rho_{0}}{\partial y}\right)-\frac{c}{2 k} u \frac{\partial u}{\partial x} \frac{\partial \rho_{0}}{\partial x} \\
& -\frac{c}{2 k} u \frac{\partial u}{\partial y} \frac{\partial \rho_{0}}{\partial y}
\end{aligned}
$$

or, equivalently,

$$
\frac{\partial \rho_{0}}{\partial t}=\left(\frac{1-\frac{c}{k}}{2}\right) u\left(\frac{\partial u}{\partial x} \frac{\partial \rho_{0}}{\partial x}+\frac{\partial u}{\partial y} \frac{\partial \rho_{0}}{\partial y}\right)+\frac{u^{2}}{2} \Delta \rho_{0} .
$$

In order to compare this with the experimental results, we substitute $u=\sqrt{\tau} v$, where $\tau$ is the reorientation characteristic time in the experiments, to obtain

$$
\frac{\partial \rho_{0}}{\partial t}=\frac{\tau}{2}\left(1-\frac{\delta}{\tau}\right) v\left(\frac{\partial v}{\partial x} \frac{\partial \rho_{0}}{\partial x}+\frac{\partial v}{\partial y} \frac{\partial \rho_{0}}{\partial y}\right)+\frac{\tau v^{2}}{2} \Delta \rho_{0} .
$$

\section{a. Radial drift [Eq. (6)]}

Equation (B8) is the backward Kolmogorov equation for the SDE system 


$$
\left\{\begin{array}{l}
d x_{t}=\frac{\tau}{2}\left(1-\frac{\delta}{\tau}\right) v\left(x_{t}, y_{t}\right) \frac{\partial v}{\partial x}\left(x_{t}, y_{t}\right) d t+\sqrt{\tau} v\left(x_{t}, y_{t}\right) d W_{t}^{1}, \\
d y_{t}=\frac{\tau}{2}\left(1-\frac{\delta}{\tau}\right) v\left(x_{t}, y_{t}\right) \frac{\partial v}{\partial y}\left(x_{t}, y_{t}\right) d t+\sqrt{\tau} v\left(x_{t}, y_{t}\right) d W_{t}^{2}
\end{array}\right.
$$

where $W^{1}$ and $W^{2}$ are independent Wiener processes. Letting $r=\sqrt{x^{2}+y^{2}}$ and applying the Itô formula, we obtain

$$
\begin{aligned}
d r_{t}= & {\left[\frac{\tau}{2}\left(1-\frac{\delta}{\tau}\right) v\left(\frac{\partial v}{\partial x} \frac{\partial r}{\partial x}+\frac{\partial v}{\partial y} \frac{\partial r}{\partial y}\right)\right.} \\
& \left.+\frac{\tau v^{2}}{2}\left(\frac{\partial^{2} r}{\partial x^{2}}+\frac{\partial^{2} r}{\partial y^{2}}\right)\right] d t \\
& +\sqrt{\tau} v\left(\frac{\partial r}{\partial x} d W_{t}^{1}+\frac{\partial r}{\partial y} d W_{t}^{2}\right)
\end{aligned}
$$

or, equivalently,

$$
\begin{aligned}
d r_{t}= & {\left[\frac{\tau}{2}\left(1-\frac{\delta}{\tau}\right) \frac{v}{r_{t}}\left(x_{t} \frac{\partial v}{\partial x}+y_{t} \frac{\partial v}{\partial y}\right)+\frac{\tau v^{2}}{r_{t}}\right] d t } \\
& +\frac{\sqrt{\tau} v}{r_{t}}\left(x_{t} d W_{t}^{1}+y_{t} d W_{t}^{2}\right)
\end{aligned}
$$

Assuming $v$ is radially symmetric, i.e., $v(x, y)=v(r)$ with $r=\sqrt{x^{2}+y^{2}}$, we have

$$
\begin{aligned}
d r_{t}= & {\left[\frac{\tau}{2}\left(1-\frac{\delta}{\tau}\right) v\left(r_{t}\right) \frac{d v}{d r}\left(r_{t}\right)+\frac{\tau v\left(r_{t}\right)^{2}}{r_{t}}\right] d t } \\
& +\frac{\sqrt{\tau} v\left(r_{t}\right)}{r_{t}}\left(x_{t} d W_{t}^{1}+y_{t} d W_{t}^{2}\right)
\end{aligned}
$$

We note that $\left(x_{t} / r_{t}\right) d W_{t}^{1}+\left(y_{t} / r_{t}\right) d W_{t}^{2}$ is the stochastic differential of the martingale

$$
B_{t}=\int_{0}^{t}\left(\frac{x_{s}}{r_{s}} d W_{s}^{1}+\frac{y_{s}}{r_{s}} d W_{s}^{2}\right)
$$

which is a Wiener process by the Lévy theorem [33] [Theorem 8.6.1] because its quadratic variation is equal to $t$. Thus, the effective radial drift is given by

$$
D(r)=\frac{\tau}{2}\left(1-\frac{\delta}{\tau}\right) v(r) \frac{d v}{d r}(r)+\frac{\tau v(r)^{2}}{r}
$$

\section{b. Density [Eq. (7)]}

The forward Kolmogorov equation corresponding to Eq. (B8) is

$$
\begin{aligned}
\frac{\partial \rho_{0}}{\partial t}= & -\frac{\tau}{2}\left(1-\frac{\delta}{\tau}\right)\left[\frac{\partial}{\partial x}\left(v \frac{\partial v}{\partial x} \rho_{0}\right)+\frac{\partial}{\partial y}\left(v \frac{\partial v}{\partial y} \rho_{0}\right)\right] \\
& +\frac{\tau}{2} \Delta\left(v^{2} \rho_{0}\right)
\end{aligned}
$$

Thus, the stationary probability density $\rho_{0}$ satisfies

$$
\begin{aligned}
- & \frac{\tau}{2}\left(1-\frac{\delta}{\tau}\right)\left[\frac{\partial}{\partial x}\left(v \frac{\partial}{\partial x} \rho_{0}\right)+\frac{\partial}{\partial y}\left(v \frac{\partial}{\partial y} \rho_{0}\right)\right] \\
& +\frac{\tau}{2} \Delta\left(v^{2} \rho_{0}\right)=0 .
\end{aligned}
$$

One can check that

$$
\rho_{0}(x, y)=\frac{1}{N v(x, y)^{1+(\delta / \tau)}},
$$

where $N$ is the normalization constant, is a positive solution of Eq. (B11).

\section{Mathematical derivation in three dimensions}

Equation (8) describes the motion of a particle in three dimensions whose speed is given by $v(x, y, z)$ and whose direction is given by an accelerated Wiener process on the surface of the unit sphere (just like in two dimensions, $e^{i \sqrt{(2 / \tau)} W_{t}}$ is an accelerated Wiener process on the unit circle). The precise form of the equations is

$$
\left\{\begin{aligned}
d x_{t} & =\frac{1}{\sqrt{\tilde{\tau}}} u\left(x_{t-\tilde{\delta}}, y_{t-\tilde{\delta}}, z_{t-\tilde{\delta}}\right) \sin \theta_{t} \cos \phi_{t} d t \\
d y_{t} & =\frac{1}{\sqrt{\tilde{\tau}}} u\left(x_{t-\tilde{\delta}}, y_{t-\tilde{\delta}}, z_{t-\tilde{\delta}}\right) \sin \theta_{t} \sin \phi_{t} d t \\
d z_{t} & =\frac{1}{\sqrt{\tilde{\tau}}} u\left(x_{t-\tilde{\delta}}, y_{t-\tilde{\delta}}, z_{t-\tilde{\delta}}\right) \cos \theta_{t} d t \\
d \theta_{t} & =\frac{1}{\tilde{\tau}} \cot \theta_{t} d t+\sqrt{\frac{2}{\tilde{\tau}}} d W_{t}^{1}, \\
d \phi_{t} & =\sqrt{\frac{2}{\tilde{\tau}}} \frac{1}{\sin \theta_{t}} d W_{t}^{2} .
\end{aligned}\right.
$$

The justification of the last two equations is the following. We start from a Wiener process on the sphere, i.e.,

$$
\left\{\begin{array}{l}
d \tilde{\theta}_{t}=\frac{1}{2} \cot \tilde{\theta}_{t} d t+d \tilde{W}_{t}^{1} \\
d \tilde{\phi}_{t}=\frac{1}{\sin \tilde{\theta}_{t}} d \tilde{W}_{t}^{2}
\end{array}\right.
$$

and rescale the time so that

$$
\left\{\begin{array}{l}
\theta_{t}=\tilde{\theta}_{2 t / \tilde{\tau}} \\
\phi_{t}=\tilde{\phi}_{2 t / \tilde{\tau}}
\end{array}\right.
$$

It is easy to show that these rescaled processes satisfy the last two equations of the above system with the new Wiener processes

$$
W_{t}^{j}=\sqrt{\frac{\tilde{\tau}}{2}} \tilde{W}_{2 t / \tilde{\tau}}^{j}, \quad j=1,2 .
$$

We follow the procedure that was used to analyze the twodimensional case. Let $\boldsymbol{x}_{t}=\left(x_{t}, y_{t}, z_{t}\right)$. In Eq. (B13), we expand $u$ about $t$ to first order in $\tilde{\delta}$ to get 


$$
\left\{\begin{array}{l}
\dot{x}_{t}=\frac{1}{\sqrt{\tilde{\tau}}}\left[u\left(\boldsymbol{x}_{t}\right)-\tilde{\delta} \frac{\partial u}{\partial x}\left(\boldsymbol{x}_{t}\right) \dot{x}_{t}-\tilde{\delta} \frac{\partial u}{\partial y}\left(\boldsymbol{x}_{t}\right) \dot{y}_{t}-\tilde{\delta} \frac{\partial u}{\partial z}\left(\boldsymbol{x}_{t}\right) \dot{z}_{t}\right] \sin \theta_{t} \cos \phi_{t}, \\
\dot{y}_{t}=\frac{1}{\sqrt{\tilde{\tau}}}\left[u\left(\boldsymbol{x}_{t}\right)-\tilde{\delta} \frac{\partial u}{\partial x}\left(\boldsymbol{x}_{t}\right) \dot{x}_{t}-\tilde{\delta} \frac{\partial u}{\partial y}\left(\boldsymbol{x}_{t}\right) \dot{y}_{t}-\tilde{\delta} \frac{\partial u}{\partial z}\left(\boldsymbol{x}_{t}\right) \dot{z}_{t}\right] \sin \theta_{t} \sin \phi_{t}, \\
\dot{z}_{t}=\frac{1}{\sqrt{\tau}}\left[u\left(\boldsymbol{x}_{t}\right)-\tilde{\delta} \frac{\partial u}{\partial x}\left(\boldsymbol{x}_{t}\right) \dot{x}_{t}-\tilde{\delta} \frac{\partial u}{\partial y}\left(\boldsymbol{x}_{t}\right) \dot{y}_{t}-\tilde{\delta} \frac{\partial u}{\partial z}\left(\boldsymbol{x}_{t}\right) \dot{z}_{t}\right] \cos \theta_{t}, \\
d \theta_{t}=\frac{1}{\tilde{\tau}} \cot \theta_{t} d t+\sqrt{\frac{2}{\tilde{\tau}}} d W_{t}^{1}, \\
d \phi_{t}=\sqrt{\frac{2}{\tilde{\tau}}} \frac{1}{\sin \theta_{t}} d W_{t}^{2} .
\end{array}\right.
$$

Solving the first three equations for $\dot{x}_{t}, \dot{y}_{t}$, and $\dot{z}_{t}$, we have

$$
\left\{\begin{array}{l}
\dot{x}_{t}=\frac{1}{\sqrt{\tau}} u\left(\boldsymbol{x}_{t}\right) \cos \phi_{t} \sin \theta_{t} \times\left[1+\frac{\tilde{\delta}}{\sqrt{\tau}}\left(\frac{\partial u}{\partial x}\left(\boldsymbol{x}_{t}\right) \cos \phi_{t} \sin \theta_{t}+\frac{\partial u}{\partial y}\left(\boldsymbol{x}_{t}\right) \sin \phi_{t} \sin \theta_{t}+\frac{\partial u}{\partial z}\left(\boldsymbol{x}_{t}\right) \cos \theta_{t}\right)\right]^{-1} \\
\dot{y}_{t}=\frac{1}{\sqrt{\tau}} u\left(\boldsymbol{x}_{t}\right) \sin \phi_{t} \sin \theta_{t} \times\left[1+\frac{\tilde{\delta}}{\sqrt{\tau}}\left(\frac{\partial u}{\partial x}\left(\boldsymbol{x}_{t}\right) \cos \phi_{t} \sin \theta_{t}+\frac{\partial u}{\partial y}\left(\boldsymbol{x}_{t}\right) \sin \phi_{t} \sin \theta_{t}+\frac{\partial u}{\partial z}\left(\boldsymbol{x}_{t}\right) \cos \theta_{t}\right)\right]^{-1} \\
\dot{z}_{t}=\frac{1}{\sqrt{\tilde{\tau}}} u\left(\boldsymbol{x}_{t}\right) \cos \theta_{t} \times\left[1+\frac{\tilde{\delta}}{\sqrt{\tilde{\tau}}}\left(\frac{\partial u}{\partial x}\left(\boldsymbol{x}_{t}\right) \cos \phi_{t} \sin \theta_{t}+\frac{\partial u}{\partial y}\left(\boldsymbol{x}_{t}\right) \sin \phi_{t} \sin \theta_{t}+\frac{\partial u}{\partial z}\left(\boldsymbol{x}_{t}\right) \cos \theta_{t}\right)\right]^{-1}
\end{array}\right.
$$

We approximate the system further, for $(\tilde{\delta} / \sqrt{\tilde{\tau}}) \ll 1$, by

$$
\left\{\begin{array}{l}
d x_{t}=\frac{1}{\sqrt{\tilde{\tau}}} u\left(\boldsymbol{x}_{t}\right) \cos \phi_{t} \sin \theta_{t} \times\left[1-\frac{\tilde{\delta}}{\sqrt{\tilde{\tau}}}\left(\frac{\partial u}{\partial x}\left(\boldsymbol{x}_{t}\right) \cos \phi_{t} \sin \theta_{t}+\frac{\partial u}{\partial y}\left(\boldsymbol{x}_{t}\right) \sin \phi_{t} \sin \theta_{t}+\frac{\partial u}{\partial z}\left(\boldsymbol{x}_{t}\right) \cos \theta_{t}\right)\right] d t \\
d y_{t}=\frac{1}{\sqrt{\tilde{\tau}}} u\left(\boldsymbol{x}_{t}\right) \sin \phi_{t} \sin \theta_{t} \times\left[1-\frac{\tilde{\delta}}{\sqrt{\tilde{\tau}}}\left(\frac{\partial u}{\partial x}\left(\boldsymbol{x}_{t}\right) \cos \phi_{t} \sin \theta_{t}+\frac{\partial u}{\partial y}\left(\boldsymbol{x}_{t}\right) \sin \phi_{t} \sin \theta_{t}+\frac{\partial u}{\partial z}\left(\boldsymbol{x}_{t}\right) \cos \theta_{t}\right)\right] d t \\
d z_{t}=\frac{1}{\sqrt{\tilde{\tau}}} u\left(\boldsymbol{x}_{t}\right) \cos \theta_{t} \times\left[1-\frac{\tilde{\delta}}{\sqrt{\tilde{\tau}}}\left(\frac{\partial u}{\partial x}\left(\boldsymbol{x}_{t}\right) \cos \phi_{t} \sin \theta_{t}+\frac{\partial u}{\partial y}\left(\boldsymbol{x}_{t}\right) \sin \phi_{t} \sin \theta_{t}+\frac{\partial u}{\partial z}\left(\boldsymbol{x}_{t}\right) \cos \theta_{t}\right)\right] d t
\end{array}\right.
$$

Let $\tilde{\delta}=c \epsilon, \tilde{\tau}=k \epsilon$. Then, the system we study is

$$
\left\{\begin{aligned}
d x_{t} & =\left(\frac{1}{\sqrt{k \epsilon}} u \cos \phi_{t} \sin \theta_{t}-\frac{c}{k} u \frac{\partial u}{\partial x} \cos ^{2} \phi_{t} \sin ^{2} \theta_{t}-\frac{c}{k} u \frac{\partial u}{\partial y} \cos \phi_{t} \sin \phi_{t} \sin ^{2} \theta_{t}-\frac{c}{k} u \frac{\partial u}{\partial z} \cos \phi_{t} \sin \theta_{t} \cos \theta_{t}\right) d t \\
d y_{t} & =\left(\frac{1}{\sqrt{k \epsilon}} u \sin \phi_{t} \sin \theta_{t}-\frac{c}{k} u \frac{\partial u}{\partial x} \sin \phi_{t} \cos \phi_{t} \sin ^{2} \theta_{t}-\frac{c}{k} u \frac{\partial u}{\partial y} \sin ^{2} \phi_{t} \sin ^{2} \theta_{t}-\frac{c}{k} u \frac{\partial u}{\partial z} \sin \phi_{t} \sin \theta_{t} \cos \theta_{t}\right) d t \\
d z_{t} & =\left(\frac{1}{\sqrt{k \epsilon}} u \cos \theta_{t}-\frac{c}{k} u \frac{\partial u}{\partial x} \cos \phi_{t} \sin \theta_{t} \cos \theta_{t}-\frac{c}{k} u \frac{\partial u}{\partial y} \sin \phi_{t} \sin \theta_{t} \cos \theta_{t}-\frac{c}{k} u \frac{\partial u}{\partial z} \cos ^{2} \theta_{t}\right) d t \\
d \theta_{t} & =\frac{1}{k \epsilon} \cot \theta_{t} d t+\sqrt{\frac{2}{k \epsilon}} d W_{t}^{1} \\
d \phi_{t} & =\sqrt{\frac{2}{k \epsilon}} \frac{1}{\sin \theta_{t}} d W_{t}^{2}
\end{aligned}\right.
$$

The backward Kolmogorov equation corresponding to the above system of SDEs is

$$
\begin{aligned}
\frac{\partial \rho}{\partial t}= & \frac{1}{k \epsilon} \frac{\partial^{2} \rho}{\partial \theta^{2}}+\frac{1}{k \epsilon \sin ^{2} \theta} \frac{\partial^{2} \rho}{\partial \phi^{2}}+\frac{1}{k \epsilon} \cot \theta \frac{\partial \rho}{\partial \theta}+\frac{1}{\sqrt{k \epsilon}} u \cos \phi \sin \theta \frac{\partial \rho}{\partial x} \\
& +\frac{1}{\sqrt{k \epsilon}} u \sin \phi \sin \theta \frac{\partial \rho}{\partial y}+\frac{1}{\sqrt{k \epsilon}} u \cos \theta \frac{\partial \rho}{\partial z} \\
& -\frac{c}{k} u\left(\frac{\partial u}{\partial x} \cos ^{2} \phi \sin ^{2} \theta+\frac{\partial u}{\partial y} \cos \phi \sin \phi \sin ^{2} \theta+\frac{\partial u}{\partial z} \cos \phi \sin \theta \cos \theta\right) \frac{\partial \rho}{\partial x} \\
& -\frac{c}{k} u\left(\frac{\partial u}{\partial x} \sin \phi \cos \phi \sin ^{2} \theta+\frac{\partial u}{\partial y} \sin ^{2} \phi \sin ^{2} \theta+\frac{\partial u}{\partial z} \sin \phi \sin \theta \cos \theta\right) \frac{\partial \rho}{\partial y} \\
& -\frac{c}{k} u\left(\frac{\partial u}{\partial x} \cos \phi \sin \theta \cos \theta+\frac{\partial u}{\partial y} \sin \phi \sin \theta \cos \theta+\frac{\partial u}{\partial z} \cos ^{2} \theta\right) \frac{\partial \rho}{\partial z}
\end{aligned}
$$


or, equivalently,

$$
\frac{\partial \rho}{\partial t}=\left(\frac{1}{\epsilon} L_{0}+\frac{1}{\sqrt{\epsilon}} L_{1}+L_{2}\right) \rho
$$

where

$$
L_{0}=\frac{1}{k}\left(\frac{\partial^{2}}{\partial \theta^{2}}+\frac{1}{\sin ^{2} \theta} \frac{\partial^{2}}{\partial \phi^{2}}+\cot \theta \frac{\partial}{\partial \theta}\right), \quad L_{1}=\frac{u}{\sqrt{k}}\left(\cos \phi \sin \theta \frac{\partial}{\partial x}+\sin \phi \sin \theta \frac{\partial}{\partial y}+\cos \theta \frac{\partial}{\partial z}\right)
$$

and

$$
\begin{aligned}
L_{2}= & -\frac{c}{k} u\left[\left(\frac{\partial u}{\partial x} \cos ^{2} \phi \sin ^{2} \theta+\frac{\partial u}{\partial y} \cos \phi \sin \phi \sin ^{2} \theta+\frac{\partial u}{\partial z} \cos \phi \sin \theta \cos \theta\right) \frac{\partial}{\partial x}\right. \\
& +\left(\frac{\partial u}{\partial x} \sin \phi \cos \phi \sin ^{2} \theta+\frac{\partial u}{\partial y} \sin ^{2} \phi \sin ^{2} \theta+\frac{\partial u}{\partial z} \sin \phi \sin \theta \cos \theta\right) \frac{\partial}{\partial y} \\
& \left.+\left(\frac{\partial u}{\partial x} \cos \phi \sin \theta \cos \theta+\frac{\partial u}{\partial y} \sin \phi \sin \theta \cos \theta+\frac{\partial u}{\partial z} \cos ^{2} \theta\right) \frac{\partial}{\partial z}\right]
\end{aligned}
$$

We expand $\rho$ in powers of $\sqrt{\epsilon}$,

$$
\rho=\rho_{0}+\sqrt{\epsilon} \rho_{1}+\epsilon \rho_{2}+\cdots
$$

Using Eq. (B16) in Eq. (B15) and equating terms of like powers of $\sqrt{\epsilon}$ gives the equations

$$
\begin{gathered}
O\left(\frac{1}{\epsilon}\right): L_{0} \rho_{0}=\frac{1}{k}\left(\frac{\partial^{2} \rho_{0}}{\partial \theta^{2}}+\frac{1}{\sin ^{2} \theta} \frac{\partial^{2} \rho_{0}}{\partial \phi^{2}}+\cot \theta \frac{\partial \rho_{0}}{\partial \theta}\right)=0, \\
O\left(\frac{1}{\sqrt{\epsilon}}\right): L_{1} \rho_{0}+L_{0} \rho_{1}=\frac{u}{\sqrt{k}} \cos \phi \sin \theta \frac{\partial \rho_{0}}{\partial x}+\frac{u}{\sqrt{k}} \sin \phi \sin \theta \frac{\partial \rho_{0}}{\partial y}+\frac{u}{\sqrt{k}} \cos \theta \frac{\partial \rho_{0}}{\partial z} \\
+\frac{1}{k} \frac{\partial^{2} \rho_{1}}{\partial \theta^{2}}+\frac{1}{k \sin ^{2} \theta} \frac{\partial^{2} \rho_{1}}{\partial \phi^{2}}+\frac{1}{k} \cot \theta \frac{\partial \rho_{1}}{\partial \theta}=0, \\
O(1): L_{2} \rho_{0}+L_{1} \rho_{1}+L_{0} \rho_{2}=\frac{\partial \rho_{0}}{\partial t} .
\end{gathered}
$$

In order to satisfy Eq. (B17), we choose $\rho_{0}=\rho_{0}(x, y, z, t)$, where we leave out a term of the form $A(x, y, z, t) \phi$ because we expect $\rho_{0}$ to be independent of $\phi$. Next, we find $\rho_{1}$ in terms of $\rho_{0}$ by solving Eq. (B18). We see that $\rho_{1}$ is the sum of the solutions to the partial differential equations (PDEs):

$$
\begin{aligned}
& \frac{u}{\sqrt{k}} \cos \phi \sin \theta \frac{\partial \rho_{0}}{\partial x}+\frac{1}{k} \frac{\partial^{2} \rho_{1}}{\partial \theta^{2}}+\frac{1}{k \sin ^{2} \theta} \frac{\partial^{2} \rho_{1}}{\partial \phi^{2}}+\frac{1}{k} \cot \theta \frac{\partial \rho_{1}}{\partial \theta}=0 \\
& \frac{u}{\sqrt{k}} \sin \phi \sin \theta \frac{\partial \rho_{0}}{\partial y}+\frac{1}{k} \frac{\partial^{2} \rho_{1}}{\partial \theta^{2}}+\frac{1}{k \sin ^{2} \theta} \frac{\partial^{2} \rho_{1}}{\partial \phi^{2}}+\frac{1}{k} \cot \theta \frac{\partial \rho_{1}}{\partial \theta}=0
\end{aligned}
$$

and

$$
\frac{u}{\sqrt{k}} \cos \theta \frac{\partial \rho_{0}}{\partial z}+\frac{1}{k} \frac{\partial^{2} \rho_{1}}{\partial \theta^{2}}+\frac{1}{k} \cot \theta \frac{\partial \rho_{1}}{\partial \theta}=0
$$

or, equivalently,

$$
\begin{aligned}
& \frac{u}{\sqrt{k}} \cos \phi \sin ^{3} \theta \frac{\partial \rho_{0}}{\partial x}+\frac{1}{k} \sin ^{2} \theta \frac{\partial^{2} \rho_{1}}{\partial \theta^{2}} \\
& +\frac{1}{k} \frac{\partial^{2} \rho_{1}}{\partial \phi^{2}}+\frac{1}{k} \sin \theta \cos \theta \frac{\partial \rho_{1}}{\partial \theta}=0, \\
& \frac{u}{\sqrt{k}} \sin \phi \sin ^{3} \theta \frac{\partial \rho_{0}}{\partial y}+\frac{1}{k} \sin ^{2} \theta \frac{\partial^{2} \rho_{1}}{\partial \theta^{2}}+\frac{1}{k} \frac{\partial^{2} \rho_{1}}{\partial \phi^{2}} \\
& \quad+\frac{1}{k} \sin \theta \cos \theta \frac{\partial \rho_{1}}{\partial \theta}=0,
\end{aligned}
$$

and

$$
\frac{1}{k} \frac{\partial^{2} \rho_{1}}{\partial \theta^{2}}+\frac{1}{k} \cot \theta \frac{\partial \rho_{1}}{\partial \theta}=-\frac{u}{\sqrt{k}} \cos \theta \frac{\partial \rho_{0}}{\partial z}
$$


The solution to the first equation is

$$
\rho_{1}=\frac{\sqrt{k} u}{2} \frac{\partial \rho_{0}}{\partial x} \sin \theta \cos \phi
$$

the solution to the second equation is

$$
\rho_{1}=\frac{\sqrt{k} u}{2} \frac{\partial \rho_{0}}{\partial y} \sin \theta \sin \phi
$$

and the solution to the third equation is

$$
\rho_{1}=\frac{\sqrt{k} u}{2} \frac{\partial \rho_{0}}{\partial z} \cos \theta
$$

so that

$\rho_{1}=\frac{\sqrt{k} u}{2}\left(\frac{\partial \rho_{0}}{\partial x} \sin \theta \cos \phi+\frac{\partial \rho_{0}}{\partial y} \sin \theta \sin \phi+\frac{\partial \rho_{0}}{\partial z} \cos \theta\right)$,

where we leave out a term of the form $A(x, y, z, t) \phi$ in order to make $\rho_{1}$ periodic in $\phi$. We could include a term that is constant in $\phi$ and $\theta$, but this would not change the final result. Equation (B19) implies that the function $\left(\partial \rho_{0} / \partial t\right)-L_{1} \rho_{1}-L_{2} \rho_{0}$ is in the range of the operator $L_{0}$. We are working in the Hilbert space defined by

$$
(u, v)=\int_{0}^{2 \pi} \int_{0}^{\pi} u v \sin \theta d \theta d \phi
$$

Note that the operator $\frac{1}{2}\left(\partial^{2} / \partial \theta^{2}\right)$ is not self-adjoint in this space; its adjoint is

$$
\frac{1}{2} \frac{\partial^{2}}{\partial \theta^{2}}+\cot \theta \frac{\partial}{\partial \theta}-\frac{1}{2}
$$

The adjoint of the operator $\frac{1}{2} \cot \theta(\partial / \partial \theta)$ is

$$
-\frac{1}{2} \cot \theta \frac{\partial}{\partial \theta}+\frac{1}{2}
$$

so $L_{0}$ is self-adjoint in this space. In particular, constants are in the kernel of $L_{0}^{*}$. Thus, we have

$$
\frac{1}{4 \pi} \int_{0}^{2 \pi} \int_{0}^{\pi}\left(\frac{\partial \rho_{0}}{\partial t}-L_{1} \rho_{1}-L_{2} \rho_{0}\right) \sin \theta d \theta d \phi=0
$$

so that

$$
\begin{aligned}
\frac{\partial \rho_{0}}{\partial t}= & \frac{1}{4 \pi} \int_{0}^{2 \pi} \int_{0}^{\pi}\left[\frac{u}{\sqrt{k}}\left(\cos \phi \sin \theta \frac{\partial}{\partial x}+\sin \phi \sin \theta \frac{\partial}{\partial y}+\cos \theta \frac{\partial}{\partial z}\right)\right. \\
& \times \frac{\sqrt{k} u}{2}\left(\frac{\partial \rho_{0}}{\partial x} \sin \theta \cos \phi+\frac{\partial \rho_{0}}{\partial y} \sin \theta \sin \phi+\frac{\partial \rho_{0}}{\partial z} \cos \theta\right) \\
& -\frac{c}{k} u\left(\left(\frac{\partial u}{\partial x} \cos ^{2} \phi \sin ^{2} \theta+\frac{\partial u}{\partial y} \cos \phi \sin \phi \sin ^{2} \theta+\frac{\partial u}{\partial z} \cos \phi \sin \theta \cos \theta\right) \frac{\partial}{\partial x}\right. \\
& +\left(\frac{\partial u}{\partial x} \sin \phi \cos \phi \sin ^{2} \theta+\frac{\partial u}{\partial y} \sin ^{2} \phi \sin ^{2} \theta+\frac{\partial u}{\partial z} \sin \phi \sin \theta \cos \theta\right) \frac{\partial}{\partial y} \\
& \left.\left.+\left(\frac{\partial u}{\partial x} \cos \phi \sin \theta \cos \theta+\frac{\partial u}{\partial y} \sin \phi \sin \theta \cos \theta+\frac{\partial u}{\partial z} \cos ^{2} \theta\right) \frac{\partial}{\partial z}\right) \rho_{0}\right] \sin \theta d \theta d \phi .
\end{aligned}
$$

Using $\int_{0}^{2 \pi} \sin \phi d \phi=\int_{0}^{2 \pi} \cos \phi d \phi=\int_{0}^{2 \pi} \sin \phi \cos \phi d \phi=0$, this simplifies to

$$
\begin{aligned}
\frac{\partial \rho_{0}}{\partial t}= & \frac{1}{4 \pi} \int_{0}^{2 \pi} \int_{0}^{\pi}\left[\frac{u}{2} \cos ^{2} \phi \sin ^{3} \theta \frac{\partial}{\partial x}\left(u \frac{\partial \rho_{0}}{\partial x}\right)+\frac{u}{2} \sin ^{2} \phi \sin ^{3} \theta \frac{\partial}{\partial y}\left(u \frac{\partial \rho_{0}}{\partial y}\right)\right. \\
& +\frac{u}{2} \cos ^{2} \theta \sin \theta \frac{\partial}{\partial z}\left(u \frac{\partial \rho_{0}}{\partial z}\right)-\frac{c}{k} u \frac{\partial u}{\partial x} \cos ^{2} \phi \sin ^{3} \theta \frac{\partial \rho_{0}}{\partial x} \\
& \left.-\frac{c}{k} u \frac{\partial u}{\partial y} \sin ^{2} \phi \sin ^{3} \theta \frac{\partial \rho_{0}}{\partial y}-\frac{c}{k} u \frac{\partial u}{\partial z} \cos ^{2} \theta \sin \theta \frac{\partial \rho_{0}}{\partial z}\right] d \theta d \phi .
\end{aligned}
$$

Using $\int_{0}^{\pi} \sin ^{3} \theta d \theta=\frac{4}{3}, \int_{0}^{\pi} \cos ^{2} \theta \sin \theta d \theta=\frac{2}{3}$, and $\int_{0}^{2 \pi} \cos ^{2} \phi d \phi=\int_{0}^{2 \pi} \sin ^{2} \phi d \phi=\pi$, we have

$$
\frac{\partial \rho_{0}}{\partial t}=\frac{u}{6} \frac{\partial}{\partial x}\left(u \frac{\partial \rho_{0}}{\partial x}\right)+\frac{u}{6} \frac{\partial}{\partial y}\left(u \frac{\partial \rho_{0}}{\partial y}\right)+\frac{u}{6} \frac{\partial}{\partial z}\left(u \frac{\partial \rho_{0}}{\partial z}\right)-\frac{c}{3 k} u \frac{\partial u}{\partial x} \frac{\partial \rho_{0}}{\partial x}-\frac{c}{3 k} u \frac{\partial u}{\partial y} \frac{\partial \rho_{0}}{\partial y}-\frac{c}{3 k} u \frac{\partial u}{\partial z} \frac{\partial \rho_{0}}{\partial z}
$$


or, equivalently,

$$
\begin{aligned}
\frac{\partial \rho_{0}}{\partial t}= & \left(\frac{1-2 \frac{c}{k}}{6}\right) u\left(\frac{\partial u}{\partial x} \frac{\partial \rho_{0}}{\partial x}+\frac{\partial u}{\partial y} \frac{\partial \rho_{0}}{\partial y}+\frac{\partial u}{\partial z} \frac{\partial \rho_{0}}{\partial z}\right) \\
& +\frac{u^{2}}{6} \Delta \rho_{0} .
\end{aligned}
$$

As in the two-dimensional case, we substitute $u=\sqrt{\tau} v$ to obtain

$$
\begin{aligned}
\frac{\partial \rho_{0}}{\partial t}= & \frac{\tau}{6}\left(1-2 \frac{\delta}{\tau}\right) v\left(\frac{\partial v}{\partial x} \frac{\partial \rho_{0}}{\partial x}+\frac{\partial v}{\partial y} \frac{\partial \rho_{0}}{\partial y}+\frac{\partial v}{\partial z} \frac{\partial \rho_{0}}{\partial z}\right) \\
& +\frac{\tau v^{2}}{6} \Delta \rho_{0} .
\end{aligned}
$$

\section{a. Radial drift}

Equation (B20) is the backward Kolmogorov equation for the SDE,

$$
\left\{\begin{array}{l}
d x_{t}=\frac{\tau}{6}\left(1-2 \frac{\delta}{\tau}\right) v\left(x_{t}\right) \frac{\partial v}{\partial x}\left(x_{t}\right) d t+\sqrt{\frac{\tau}{3}} v\left(x_{t}\right) d W_{t}^{1}, \\
d y_{t}=\frac{\tau}{6}\left(1-2 \frac{\delta}{\tau}\right) v\left(x_{t}\right) \frac{\partial v}{\partial y}\left(x_{t}\right) d t+\sqrt{\frac{\tau}{3}} v\left(x_{t}\right) d W_{t}^{2}, \\
d z_{t}=\frac{\tau}{6}\left(1-2 \frac{\delta}{\tau}\right) v\left(x_{t}\right) \frac{\partial v}{\partial z}\left(x_{t}\right) d t+\sqrt{\frac{\tau}{3}} v\left(x_{t}\right) d W_{t}^{3},
\end{array}\right.
$$

where $W^{1}, W^{2}$, and $W^{3}$ are independent Wiener processes. Letting $r=\sqrt{x^{2}+y^{2}+z^{2}}$ and applying the Itô formula, we obtain

$$
\begin{aligned}
d r_{t}= & {\left[\frac{\tau}{6}\left(1-2 \frac{\delta}{\tau}\right) v\left(\frac{\partial v}{\partial x} \frac{\partial r}{\partial x}+\frac{\partial v}{\partial y} \frac{\partial r}{\partial y}+\frac{\partial v}{\partial z} \frac{\partial r}{\partial z}\right)\right.} \\
& \left.+\frac{\tau v^{2}}{6}\left(\frac{\partial^{2} r}{\partial x^{2}}+\frac{\partial^{2} r}{\partial y^{2}}+\frac{\partial^{2} r}{\partial z^{2}}\right)\right] d t \\
& +\sqrt{\frac{\tau}{3}} v\left(\frac{\partial r}{\partial x} d W_{t}^{1}+\frac{\partial r}{\partial y} d W_{t}^{2}+\frac{\partial r}{\partial z} d W_{t}^{3}\right)
\end{aligned}
$$

or, equivalently,

$$
\begin{aligned}
d r_{t}= & {\left[\frac{\tau}{6}\left(1-2 \frac{\delta}{\tau}\right) \frac{v}{r_{t}}\left(x_{t} \frac{\partial v}{\partial x}+y_{t} \frac{\partial v}{\partial y}+z_{t} \frac{\partial v}{\partial z}\right)+\frac{\tau v^{2}}{3 r_{t}}\right] d t } \\
& +\sqrt{\frac{\tau}{3}} \frac{v}{r_{t}}\left(x_{t} d W_{t}^{1}+y_{t} d W_{t}^{2}+z_{t} d W_{t}^{3}\right) .
\end{aligned}
$$

Assuming $v$ is radially symmetric, i.e., $v(x, y, z)=v(r)$, we have

$$
\begin{aligned}
d r_{t}= & {\left[\frac{\tau}{6}\left(1-2 \frac{\delta}{\tau}\right) v\left(r_{t}\right) \frac{d v}{d r}\left(r_{t}\right)+\frac{\tau v\left(r_{t}\right)^{2}}{3 r_{t}}\right] d t } \\
& +\sqrt{\frac{\tau}{3}} \frac{v\left(r_{t}\right)}{r_{t}}\left(x_{t} d W_{t}^{1}+y_{t} d W_{t}^{2}+z_{t} d W_{t}^{3}\right) .
\end{aligned}
$$

We note that $\frac{x_{t}}{r_{t}} d W_{t}^{1}+\frac{y_{t}}{r_{t}} d W_{t}^{2}+\frac{z_{t}}{r_{t}} d W_{t}^{3}$ is the stochastic differential of

$$
B_{t}=\int_{0}^{t}\left(\frac{x_{s}}{r_{s}} d W_{s}^{1}+\frac{y_{s}}{r_{s}} d W_{s}^{2}+\frac{z_{s}}{r_{s}} d W_{s}^{3}\right),
$$

which is a Wiener process because its quadratic variation is equal to $t$. Thus, the radial drift is given by

$$
D(r)=\frac{\tau}{6}\left(1-2 \frac{\delta}{\tau}\right) v(r) \frac{d v}{d r}(r)+\frac{\tau v(r)^{2}}{3 r} .
$$

\section{b. Density [Eq. (9)]}

The forward Kolmogorov equation corresponding to Eq. (B20) is

$$
\begin{aligned}
\frac{\partial \rho_{0}}{\partial t}= & -\frac{\tau}{6}\left(1-2 \frac{\delta}{\tau}\right)\left[\frac{\partial}{\partial x}\left(v \frac{\partial v}{\partial x} \rho_{0}\right)+\frac{\partial}{\partial y}\left(v \frac{\partial v}{\partial y} \rho_{0}\right)\right. \\
& \left.+\frac{\partial}{\partial z}\left(v \frac{\partial v}{\partial z} \rho_{0}\right)\right]+\frac{\tau}{6} \Delta\left(v^{2} \rho_{0}\right) .
\end{aligned}
$$

Thus, by equating the right-hand side to zero, we find that the stationary probability density $\rho_{0}$ (if it exists) is

$$
\rho_{0}(x, y, z)=\frac{1}{M v(x, y, z)^{1+2(\delta / \tau)}},
$$

where $M$ is the normalization constant.

[1] J. A. Shapiro, Thinking About Bacterial Populations as Multicellular Organisms, Annu. Rev. Microbiol. 52, 81 (1998).

[2] H. C. Berg, E. coli in Motion (Springer Science \& Business Media, New York, NY, 2004).

[3] G. M. Viswanathan, M. G. E. Da Luz, E. P. Raposo, and H. E. Stanley, The Physics of Foraging: An Introduction to Random Searches and Biological Encounters (Cambridge University Press, Cambridge, England, 2011).

[4] J. K. Parrish, S. V. Viscido, and D. Grünbaum, SelfOrganized Fish Schools: An Examination of Emergent Properties, Biol. Bull. 202, 296 (2002).

[5] M. Moussaïd, D. Helbing, S. Garnier, A. Johansson, M. Combe, and G. Theraulaz, Experimental Study of the Behavioural Mechanisms Underlying Self-Organization in Human Crowds, Proc. Roy. Soc. B: Biol. Sci. 276, 2755 (2009).

[6] E. Bonabeau, M. Dorigo, and G. Theraulaz, Inspiration for Optimization from Social Insect Behaviour, Nature (London) 406, 39 (2000).

[7] J. Halloy, G. Sempo, G. Caprari, C. Rivault, M. Asadpour, F. Tâche, I. Said, V. Durier, S. Canonge, J. M. Amé et al., Social Integration of Robots into Groups of Cockroaches to Control Self-Organized Choices, Science 318, 1155 (2007). 
[8] E. Şahin and A. Winfield, Special Issue on Swarm Robotics, Swarm Intelligence 2, 69 (2008).

[9] M. Brambilla, E. Ferrante, M. Birattari, and M. Dorigo, Swarm Robotics: A Review from the Swarm Engineering Perspective, Swarm Intelligence 7, 1 (2013).

[10] M. Rubenstein, A. Cornejo, and R. Nagpal, Programmable Self-Assembly in a Thousand-Robot Swarm, Science 345, 795 (2014).

[11] J. Werfel, K. Petersen, and R. Nagpal, Designing Collective Behavior in a Termite-Inspired Robot Construction Team, Science 343, 754 (2014).

[12] F. Schweitzer, Brownian Agents and Active Particles (Springer, Berlin, 2003).

[13] T. Vicsek and A. Zafeiris, Collective Motion, Phys. Rep. 517, 71 (2012).

[14] R. M. Macnab and D. E. Koshland, The Gradient-Sensing Mechanism in Bacterial Chemotaxis, Proc. Natl. Acad. Sci. U.S.A. 69, 2509 (1972).

[15] T. Vicsek, A. Czirók, E. Ben-Jacob, I. Cohen, and O. Shochet, Novel Type of Phase Transition in a System of Self-Driven Particles, Phys. Rev. Lett. 75, 1226 (1995).

[16] M. Dorigo, V. Trianni, E. Şahin, R. Groß, T. H. Labella, G. Baldassarre, S. Nolfi, J.-L. Deneubourg, F. Mondada, D. Floreano, and L. M. Gambardella, Evolving Self-Organizing Behaviors for a Swarm-Bot, Autonomous Robots 17, 223 (2004).

[17] O. Chepizhko and F. Peruani, Diffusion, Subdiffusion, and Trapping of Active Particles in Heterogeneous Media, Phys. Rev. Lett. 111, 160604 (2013).

[18] J. Palacci, S. Sacanna, A. P. Steinberg, D. J. Pine, and P. M. Chaikin, Living Crystals of Light-Activated Colloidal Surfers, Science 339, 936 (2013).

[19] S. J. Ebbens and J. R. Howse, In Pursuit of Propulsion at the Nanoscale, Soft Matter 6, 726 (2010).

[20] G. Volpe, S. Gigan, and G. Volpe, Simulation of the Active Brownian Motion of a Microswimmer, Am. J. Phys. 82, 659 (2014).
[21] J. L. Souman, I. Frissen, M. N. Sreenivasa, and M. O. Ernst, Walking Straight into Circles, Curr. Biol. 19, 1538 (2009).

[22] J. R. Howse, R. A. L. Jones, A. J. Ryan, T. Gough, R. Vafabakhsh, and R. Golestanian, Self-Motile Colloidal Particles: From Directed Propulsion to Random Walk, Phys. Rev. Lett. 99, 048102 (2007).

[23] F. Peruani and L. G. Morelli, Self-Propelled Particles with Fluctuating Speed and Direction of Motion in Two Dimensions, Phys. Rev. Lett. 99, 010602 (2007).

[24] Elisa-3, http://www.gctronic.com/doc/index.php/Elisa-3.

[25] J.E. Segall, S. M. Block, and H.C. Berg, Temporal Comparisons in Bacterial Chemotaxis, Proc. Natl. Acad. Sci. U.S.A. 83, 8987 (1986).

[26] Y. Chen, J.H. Lü, and X. H. Yu, Robust Consensus of Multi-agent Systems with Time-Varying Delays in Noisy Environment, Sci. China Tech. Sci. 54, 2014 (2011).

[27] E. Forgoston and I. B. Schwartz, Delay-Induced Instabilities in Self-Propelling Swarms, Phys. Rev. E 77, 035203 (2008).

[28] Y. Sun, W. Lin, and R. Erban, Time Delay Can Facilitate Coherence in Self-Driven Interacting-Particle Systems, Phys. Rev. E 90, 062708 (2014).

[29] G. Pesce, A. McDaniel, S. Hottovy, J. Wehr, and G. Volpe, Stratonovich-to-Itô Transition in Noisy Systems with Multiplicative Feedback, Nat. Commun. 4, 2733 (2013).

[30] P. E. Kloeden and E. Platen, Numerical Solution of Stochastic Differential Equations (Springer Science \& Business Media, Heidelberg, 1992).

[31] R. Großmann, F. Peruani, and M. Bär, A Geometric Approach to Self-Propelled Motion in Isotropic \& Anisotropic Environments, Eur. Phys. J. Spec. Top. 224, 1377 (2015).

[32] G. A. Pavliotis and A. M. Stuart, Multiscale Methods (Springer, Heidelberg, Germany, 2008).

[33] B. Øksendal, Stochastic Differential Equations (Springer, Heidelberg, Germany, 2007). 